\title{
Active Flow Control at Low Reynolds Numbers on A NACA 0015 Airfoil
}

\author{
LaTunia Pack Melton, Judith Hannon†, Chung-Sheng Yao† and Jerome Harris ${ }^{\ddagger}$ \\ NASA Langley Research Center, Hampton, Virginia, United States of America
}

\begin{abstract}
Results from a low Reynolds number wind tunnel experiment on a NACA 0015 airfoil with a $30 \%$ chord trailing edge flap tested at deflection angles of $0^{\circ}, 20^{\circ}$, and $40^{\circ}$ are presented and discussed. Zero net mass flux periodic excitation was applied at the flap shoulder to control flow separation for flap deflections larger than $0^{\circ}$. The primary objective of the experiment was to compare force and moment data obtained from integrating surface pressures to data obtained from a 5-component strain-gage balance in preparation for additional three-dimensional testing of the model. To achieve this objective, active flow control is applied at an angle of attack of $6^{\circ}$ where published results indicate that oscillatory momentum coefficients exceeding $1 \%$ are required to delay separation. Periodic excitation with an oscillatory momentum coefficient of $1.5 \%$ and a reduced frequency of 0.71 caused a significant delay of separation on the airfoil with a flap deflection of $20^{\circ}$. Higher momentum coefficients at the same reduced frequency were required to achieve a similar level of flow attachment on the airfoil with a flap deflection of $40^{\circ}$. There was a favorable comparison between the balance and integrated pressure force and moment results.
\end{abstract}

\section{Nomenclature}

$c \quad$ airfoil chord $\left(\delta_{f}=0^{\circ}\right), \mathrm{m}$

$C_{\mu} \quad$ excitation momentum coefficient, $\equiv<J^{\prime}>/ c q$

$C_{d p} \quad$ pressure drag coefficient

$C_{D} \quad$ total drag coefficient

$C_{L} \quad$ lift coefficient

$C_{L, \max }$ maximum lift coefficient

$C_{p} \quad$ pressure coefficient, $\equiv\left(P-P_{s}\right) / q$

$f \quad$ frequency, $\mathrm{Hz}$

$F^{+} \quad$ reduced frequency, $\equiv\left(f x_{s p}\right) / U_{\infty}$

$h \quad$ slot width, mm

$J^{\prime} \quad$ oscillatory momentum at slot exit, $\equiv \rho h u_{j}^{\prime 2}$

$P \quad$ pressure

$P_{s} \quad$ static pressure

$q \quad$ free stream dynamic pressure, $\equiv 1 / 2 \rho U_{\infty}^{2}$

$\operatorname{Re}_{c} \quad$ chord Reynolds number, $\equiv U_{\infty} c / \nu$

$s \quad$ airfoil span, $\mathrm{m}$

$t \quad$ time, $\mathrm{s}$

$U, u \quad$ average and fluctuating streamwise velocity, $\mathrm{m} / \mathrm{s}$

$x / c \quad$ normalized streamwise location

$x_{s p} \quad$ distance from actuator to trailing edge

y spanwise location

$\alpha \quad$ angle of attack, deg

$\delta_{f} \quad$ flap deflection, deg

$\xi \quad \mathrm{x}$ rotated $6^{\circ}$

\footnotetext{
${ }^{*}$ Research Scientist, Flow Physics and Control Branch, MS 170, Senior Member AIAA

$\dagger$ Research Scientist, Flow Physics and Control Branch, MS 170

$\ddagger$ Optical Technician, Flow Physics and Control Branch, MS 170
} 
$\nu \quad$ kinematic viscosity

$\zeta \quad$ y rotated $6^{\circ}$

$\rho \quad$ density

$\phi \quad$ phase of excitation cycle

\section{A. Abbreviations}

$2 D \quad$ two-dimensional

$3 D \quad$ three-dimensional

$A F C$ active flow control

$B M \quad$ burst modulation

$L E \quad$ leading edge

$T E \quad$ trailing edge

\section{B. Subscripts}

$\begin{array}{ll}\mathrm{j} & \text { conditions at excitation slot } \\ \mathrm{M} & \text { modulation } \\ \infty & \text { free stream conditions }\end{array}$

\section{Superscript}

root mean square of fluctuating value

\section{Introduction}

Active separation control is considered one of the enabling technologies for the next generation of hybrid wing body vehicles. For this reason, the National Aeronautics and Space Administration's Subsonic Fixed Wing Project funds both internal and external research in this area. This research is fundamental, long-term research focused on obtaining a better understanding of the physics governing active separation control. Our internal research is focused on examining the effects of the excitation frequency and amplitude of periodic excitation on two-dimensional (2D) and three-dimensional (3D) flowfields. This will be accomplished through a series of wind tunnel experiments on a model with a NACA 0015 cross-section.

One motivation for the use of periodic excitation as a method of controlling separation is the reduction in momentum required to control separation when compared to steady momentum transfer methods. Seifert et al. ${ }^{1}$ reported a one to two order of magnitude reduction in momentum requirements at low Reynolds numbers when using periodic excitation as compared to steady blowing at the leading edge of a NACA 0015 airfoil. Later, Seifert and $\mathrm{Pack}^{2}$ reported similar reductions in momentum requirements on a NACA 0015 at flight Reynolds numbers. Periodic excitation is effective for controlling separation when introduced just upstream of the separation location. The vortical structures introduced by the 2D excitation are used to enhance the mixing between the high momentum fluid of the separated shear layer and the low momentum fluid in the separated region. Introduction of the excitation at frequencies that correspond to the natural instabilities in the separating shear layer is the reason for the efficiency of periodic excitation when compared to steady methods of separation control. Periodic excitation applied near the leading edge of an airfoil delays stall and increases the maximum lift $\left(C_{L, \max }\right)$ generated by the airfoil. Periodic excitation applied at the flap shoulder delays or prevents separation on the deflected flap of the airfoil, resulting in improved flap efficiency. Additional information on the technology can be found in the review papers of Greenblatt and Wygnanski ${ }^{3}$ and Seifert et al. ${ }^{4}$.

The efficiency of periodic excitation as well as the perceived ease of implementation when compared to steady blowing or suction are reasons for continued research to understand the effects of model geometry ( i.e., flap deflection angle, slot location, and surface curvature) and excitation frequency and amplitude. 
Efficiency is also important when controlling separation on an airfoil or wing with multiple slot locations. In reference 5, the authors report a sensitivity to phase when combining excitations from two closely spaced sources around the flap shoulder of a supercritical airfoil. The lift increment of the airfoil was maximized when the relative phase between the excitations was $\pm 30^{\circ}$. The single frequency selected to combine the two excitations corresponded to the frequency that was most effective for separation control when each actuator was operated individually. The optimal phase angle for combining excitations from two closely spaced actuators was validated at a higher Reynolds number on a larger version of the airfoil. ${ }^{6}$ Recently, Greenblatt ${ }^{7}$ combined leading edge and flap shoulder excitations on a semi-span model with a NACA 0015 cross-section and demonstrated that the maximum lift increment occurred when the relative phase between the two excitations was approximately $210^{\circ}$. The two excitations were combined using the frequency that was most effective for leading edge separation control. Particle Image Velocimetry (PIV) data revealed that at a phase angle of $210^{\circ}$, the oscillating shear layer was closer to the surface during the suction portion of the cycle of the flap shoulder excitation. ${ }^{7}$ Based on the two models studied, the optimal phase angle for combining two excitations is dependent upon the distance between the excitation slots, the excitation frequency, and the freestream velocity.

The semi-span model, with a NACA 0015 cross-section, designed and tested by Greenblatt ${ }^{7}$ was used for the current investigation. While most of the research planned for this model will focus on the 3D configuration, the $2 \mathrm{D}$ experiments presented in this paper were performed to incorporate a balance into the system. The $2 \mathrm{D}$ experiment also provided the opportunity to gather additional data that will be useful in selecting and locating additional trailing edge flap actuators and to study the transients of flow separation and attachment in preparation for planned closed-loop control research. The results of Greenblatt and Washburn ${ }^{8}$ suggest $^{2}$ that there is a need for additional and/or different actuators for improved control authority on the threedimensional model configuration. The balance measurements will provide an independent measurement of the forces and moments for comparison to the surface pressure interpolation method described in reference 9 and used on the 3D configuration. In this paper we focus on controlling separation on the deflected flap of the airfoil at an angle of attack, $\alpha$, greater than 0 degrees where the momentum requirements for controlling separation are considered relatively high compared to those required at lower angles of attack. Force and moment data and Particle Image Velocimetery (PIV) data will be presented to illustrate the effects that frequency and amplitude have on separation control.

\section{Experiment Description}

\section{A. Wind Tunnel Description}

The experiments were conducted in the NASA Langley Research Center (LaRC) 2 Foot x 3 Foot Low Speed Wind Tunnel. The 10:1 contraction ratio closed-circuit tunnel has a test section that is $0.61 \mathrm{~m}$ high x 0.91 $\mathrm{m}$ wide by $6.1 \mathrm{~m}$ long. The tunnel turbulence intensities are approximately $0.1 \%$ between $0.1<f<400 \mathrm{~Hz}$. Additional information about the wind tunnel can be found in reference 10. The maximum velocity of the tunnel is approximately $45 \mathrm{~m} / \mathrm{s}$. The data that will be presented were acquired at a chord Reynolds number of $0.25 \times 10^{6}$, corresponding to a tunnel velocity of approximately $13 \mathrm{~m} / \mathrm{s}$.

\section{B. Model Description}

The model tested is a $0.3048 \mathrm{~m}$ chord (c), $0.6096 \mathrm{~m}$ span (s) airfoil that Greenblatt ${ }^{7}$ tested in a 3D configuration in the $0.711 \mathrm{~m} \times 1.016 \mathrm{~m}$ test section of the LaRC Basic Aerodynamics Research Tunnel. The airfoil has a $30 \%$ chord trailing edge flap divided into three sections that can be tested at flap deflections, $\delta_{f}$, from $-10^{\circ}$ to $40^{\circ}$ in $10^{\circ}$ increments. The results presented are with all sections of the flap at the same deflection angle $\left(\delta_{f}=0^{\circ}, 20^{\circ}\right.$, or $\left.40^{\circ}\right)$. Internal chambers in the model connected to slots in the airfoil surface at the leading edge and flap shoulder of the model are used to introduce periodic excitation for separation control. The $0.5 \mathrm{~mm}$ wide leading edge slot and $0.76 \mathrm{~mm}$ wide flap shoulder slot were designed so that the air exiting the slot would be nearly tangential to the model surface. In the case of the flap shoulder slot, the excitation is nearly tangential when $\delta_{f}=0^{\circ}$. The model has four rows of streamwise pressure taps at spanwise $(\mathrm{y} / \mathrm{s})$ locations of $0.17,0.5,0.83$, and 0.99 and four rows of spanwise pressure taps at streamwise $(\mathrm{x} / \mathrm{c})$ locations of $0.005,0.3,0.705$, and 1.0. There are three unsteady pressure transducers in the model's internal cavities 
and 12 unsteady pressure transducers connected to orifices on the model upper surface located at $\mathrm{x} / \mathrm{c}=0.005$, $0.30,0.705$, and 1.0 at $\mathrm{y} / \mathrm{s}$ locations of $0.163,0.496$, and 0.829 . The unsteady pressure voltage signals and balance voltage signals were low-pass filtered at $5 \mathrm{kHz}$ and sampled at a rate of $12.8 \mathrm{kHz}$.

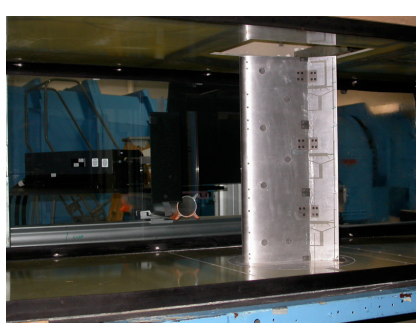

(a) Vertical model installation lower surface view.

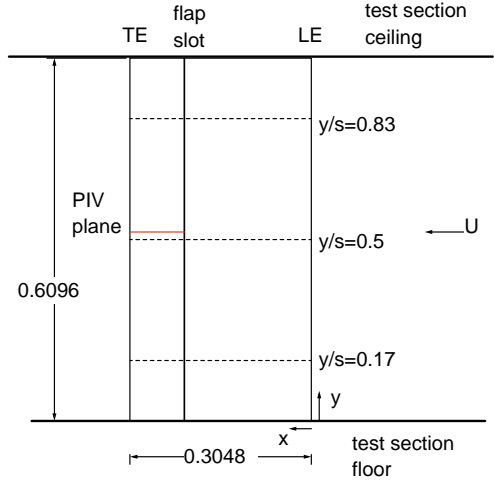

(b) Schematic of model upper surface.

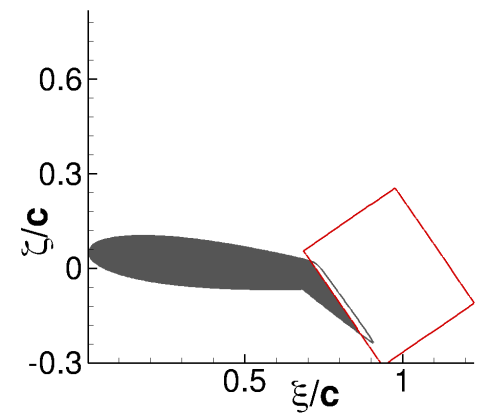

(c) PIV region definition.

Figure 1. Model description for installation in NASA LaRC 2 Foot $x 3$ Foot Wind Tunnel.

The model is oriented vertically in the tunnel for this $2 \mathrm{D}$ test as shown in figures 1 (a) and 1(b). In preparation for additional testing of the 3D configuration of the model, the model was mounted to a 5-component external strain gage balance. The General Aviation Circulation Control (GACC) balance, with characteristics shown in Table 1, was designed for a vertically mounted model. ${ }^{11}$ To prevent fouling, there is a $2.5 \mathrm{~mm}$ gap between the model and the ceiling of the tunnel. The balance chosen for the 2D tests limited the maximum Reynolds number of the experiment. The balance that will be used for follow-on 3D testing has higher load and pitching moment limits so the Reynolds number range of the tests will not be limited by the balance.

\begin{tabular}{|l|l|l|}
\hline Force/Moment & Limit & Accuracy, \% Full Scale \\
\hline \hline Normal & $100 \mathrm{lb}$ & 0.04 \\
\hline Axial & $5 \mathrm{lb}$ & 0.39 \\
\hline Pitching Moment & $400 \mathrm{in}-\mathrm{lb}$ & 0.12 \\
\hline Rolling Moment & $1200 \mathrm{in}-\mathrm{lb}$ & 0.07 \\
\hline Yawing Moment & $40 \mathrm{in}-\mathrm{lb}$ & 1.64 \\
\hline
\end{tabular}

Table 1. GACC Balance limits

\section{AFC Actuators}

Excitation is provided by two externally mounted electromagnetic actuators. The actuators are connected to the model's leading edge and flap shoulder slots by manifolds that attach to the turntable of the model. Bench-top calibrations using a hot-wire were performed prior to the wind tunnel test to determine the velocity fluctuations produced by each actuator. A sine wave with a frequency in the range of $50 \mathrm{~Hz}$ to $400 \mathrm{~Hz}$ is used to drive the actuators. Dynamic pressure transducers located in the leading and trailing edge slot cavities are used to correlate the velocity perturbations produced during bench-top testing to those produced during wind tunnel testing. The actuators are capable of producing peak velocities in excess of $50 \mathrm{~m} / \mathrm{s}$. External actuation introduced from one side of the model resulted in a reduction in the spanwise uniformity of the excitation. The excitation was strongest near the model endplate and the non-uniformity was more pronounced as the excitation frequency increased. Although the actuators and manifolds used for this experiment differ from those used by Greenblatt and Washburn, ${ }^{8}$ the trends they observed in terms of spanwise uniformity as a function of frequency are similar to the ones observed during this investigation. The 
oscillatory momentum coefficient $\left(C_{\mu}\right)$ values that will be presented are based on the actuator calibrations performed at $\mathrm{y} / \mathrm{s}=0.5$.

\section{Particle Image Velocimetry}

A commercially available high speed Particle Image Velocimetry (PIV) system was used during the test. The system consists of a high speed laser and a 1280 x 1024 camera with an internal intensifier. The maximum frame rate of the camera is approximately $1 \mathrm{kHz}$ in single exposure mode and approximately $500 \mathrm{~Hz}$ in double exposure mode. The internal memory of the camera limits an acquisition to a little more than 1600 image pairs. A $105 \mathrm{~mm}$ macro lens was used with the camera, and the field of view was $135 \mathrm{~mm} \times 107 \mathrm{~mm}$ for the $\delta_{f}=40^{\circ}$ data and $98 \mathrm{~mm} \times 78 \mathrm{~mm}$ for the $\delta_{f}=20^{\circ}$ data. For most of the data presented, a 32 pixel x 32 pixel $\left(3.38 \mathrm{~mm} \times 3.34 \mathrm{~mm}\right.$ for $\delta_{f}=40^{\circ}$ and $2.45 \mathrm{~mm}$ x $2.44 \mathrm{~mm}$ for $\left.\delta_{f}=20^{\circ}\right)$ interrogation window is used with a maximum overlap in the horizontal and vertical direction of $50 \%$. The PIV region for $\delta_{f}=40^{\circ}$ is shown in figure $1(\mathrm{c})$. The $\delta_{f}=40^{\circ} \mathrm{PIV}$ data were acquired before the $\delta_{f}=20^{\circ} \mathrm{PIV}$ data and the field of view was reduced after completing the measurements of the first configurations to improve the signal to noise ratio near the flap shoulder. To avoid possible damage to the camera CCD, the laser was aligned so that the light sheet was almost parallel to the flap of the model. This arrangement provides user control over the intensity of the laser light sheet that is permitted to impinge the model, and therefore provides control over the intensity of the light exposed to the camera sensor. The camera was located above the test section and was rotated to be at the same angle as the flap of the model to increase the amount of the flowfield above the flap surface included in each image. All of the PIV data were acquired at $\mathrm{y} / \mathrm{s}=0.52$ slightly above the centerline row of streamwise pressure taps as shown in figure 1(b). The flowfield was seeded with olive oil using a single-jet atomizer that generated particles approximately a micron in size. The baseline data presented were acquired at rates near $500 \mathrm{~Hz}$ and 1600 image pairs were used to compute the mean values presented. The data acquired when $F^{+}=0.71$ were acquired phase locked to the excitation, i.e. $100 \mathrm{~Hz}$ sample rate, and $300\left(\delta_{f}=40^{\circ}\right)$ or $400\left(\delta_{f}=20^{\circ}\right)$ image pairs were averaged to compute the mean value at each phase of the excitation cycle. The data presented when using the $F^{+}=0.36$ excitation were acquired at a sample rate of $400 \mathrm{~Hz}$ and each phase of the excitation is an average of 200 image pairs. The data presented when using burst modulation is an average of 200 image pairs that were sampled at a rate eight times the modulation frequency. A cross-correlation algorithm was used to process the PIV data. Additional analysis of the PIV results is required before quantitative information can be obtained from the data.

\section{E. Wake Rake}

A wake rake located 4.5 chord lengths downstream of the midchord of the model was used to survey the wake. Most of the wake surveys were performed using a rake at a fixed spanwise location, $\mathrm{y} / \mathrm{s}=0.5$, that has 31 total probes with a probe spacing of $17.78 \mathrm{~mm}$. Two pressure orifices on opposite tunnel walls, aligned with the wake rake total probes, were averaged to determine wake static pressure. This type of wake rake enabled the wake to be surveyed with only a few moves of the wake rake. However, the total drag values computed from data using this wake rake were lower than the total drag from balance data and the pressure drag computed from surface static pressures. Based on the recommendations in reference 12 and results from a recent experiment ${ }^{13}$ in the facility, a second wake rake was used during the experiment. This wake rake with six total probes spaced $25.4 \mathrm{~mm}$ apart in the spanwise direction, y, enabled the spanwise variations in total drag to be measured and enabled the static pressure in the wake to be measured. The time required to acquire a wake survey increased because the wake rake had to be moved across the entire wake of the model. A limited amount of data will be presented from this wake rake.

\section{F. Experimental Uncertainty}

The method described in reference 14 is used to compute uncertainties of the data. The chord Reynolds number, $R e_{c}$ is accurate to within $3 \%$. The oscillatory momentum coefficient, $C_{\mu}$, is accurate to within $25 \%$ (partly due to slot width uncertainties and partly due to the calibration uncertainties). The dynamic pressure is accurate to within $\pm 0.65 \mathrm{~Pa}$. 


\section{Discussion}

\section{A. Baseline Flow Field}

Prior to the application of active flow control from either excitation slot, the baseline flow field was examined. Since a primary objective of the test is to compare balance force and moment data with integrated values from surface pressures, data are presented comparing the two methods. Figure 2(a) presents the variation of lift with angle of attack data for the straight airfoil $\left(\delta_{f}=0^{\circ}\right)$. The lift curves for the three inboard spanwise $(\mathrm{y} / \mathrm{s})$ locations where nominally $2 \mathrm{D}$ flow is expected are shown. The pressure taps located at $\mathrm{y} / \mathrm{s}=0.99$ (not shown) are near the gap between the airfoil and the tunnel ceiling and 3D effects are evident in the pressure distributions. The values of $C_{L}$ calculated by integrating surface pressures are uniform between $\mathrm{y} / \mathrm{s}=0.17$ and $\mathrm{y} / \mathrm{s}=0.83$ prior to stall. As stall is approached, some differences are observed in the stall characteristics of the flowfield. Lift data obtained using the pressure taps at $\mathrm{y} / \mathrm{s}=0.5$ indicate at that location the airfoil has a lower $C_{L, \max }$, stalls sooner, and has lower lift values post stall compared to the other two spanwise locations and the balance data. The values of $C_{L}$ obtained by integrating pressures at different spanwise locations are similar prior to stall. For the other flap deflections $\left(\delta_{f}=20^{\circ}\right.$ and $\delta_{f}=40^{\circ}$ not shown) considered, the balance lift results compare reasonably well with the lift values obtained by integrating the surface pressures. There is slightly more variation in lift as a function of span for the $\delta_{f}=20^{\circ}$ and $\delta_{f}=40^{\circ}$ cases prior to stall, probably due to the separated flow on the flap of the model.

Pressure drag computed by integrating surface pressures and total drag measured by the balance are compared in Fig. 2(b). The total drag measured by the balance is higher than $C_{d p}$ because of the skin friction drag that is also measured by the balance. There is fairly good agreement between the pressure drag for the three spanwise locations provided in figure 2(b). Prior to airfoil stall, the largest differences in $C_{d p}$ values occurs between $\alpha=6^{\circ}$ and $\alpha=13^{\circ}$. Post stall trends are similar to the lift data of figure 2(a). The comparisons of drag performed at flap deflections of $20^{\circ}$ and $40^{\circ}$ (not shown) are similar to the comparison at $\delta_{f}=0^{\circ}$. Wake surveys were also performed to determine total drag. The data of figure 2(c) illustrates that using the wake rake at a fixed $\mathrm{y} / \mathrm{s}$ location with static pressure measured on the tunnel sidewall resulted in drag measurements for angles of attack above $6^{\circ}$ that were not comparable to the total drag measured by the balance. The wake rake (wake rake 2 in figure 2(c)) that enabled static pressures to be measured within the wake did a better job of measuring total drag. The spanwise variation of $C_{D}$ was also obtained using this wake rake and illustrated that for the Reynolds numbers of this test, wake surveys at more than one y location improved the accuracy of the drag measurements using the wake survey method. The $C_{D}$ values, obtained using wake rake 2 , shown in figure 2 (c) were computed from data acquired with the wake rake fixed at a single $\mathrm{y} / \mathrm{s}$ location. Note, wake rake 2 has six total probes with a $25.4 \mathrm{~mm}$ spacing in y. The data presented is the average total drag in the interval $0.42<y / s<0.63$.

Figure 2(d) illustrates the differences in pitching moment between balance and integrated pressure measurements. The largest difference between balance and integrated pressure results occurs when comparing pitching moment. Some of the differences are due to the higher uncertainty in the measurement of pitching moment using the balance.

The post-stall $C_{p}$ data (not shown) for the airfoil with $\delta_{f}=0^{\circ}$ as well as the higher flap deflections indicate the model stalled as a result of laminar leading edge (LE) separation rather than trailing edge separation. For this reason, the results presented focus on trailing edge flap separation control at angle of attack settings below $C_{L, \max }$. The flowfield where the excitation is being introduced should be turbulent and thus reduce the contributions of transitional effects to the overall effectiveness of the control. Leading edge control was revisited later in the experiment and a transition strip comprised of tape was added near the stagnation point to prevent laminar LE separation. These data are not presented in the paper, however some of the trailing edge flap results included are with this strip on the model.

Streamwise velocity contours from PIV measurements acquired along the flap of the model at $\mathrm{y} / \mathrm{s}=0.52$ are presented in figure 3 for $\delta_{f}=20^{\circ}$ and $\delta_{f}=40^{\circ}$ to illustrate the differences in the baseline flowfields that will be controlled. These figures indicate that the separation region is much larger with the larger flap deflection. Note that the PIV data is in airfoil coordinates and is normalized by the maximum velocity determined from the PIV data. The data of figure 3 are the mean values computed from 1600 image pairs acquired at sample rates of $500 \mathrm{~Hz}$ and $474 \mathrm{~Hz}$ for the $\delta_{f}=20^{\circ}$ and $\delta_{f}=40^{\circ}$ data, respectively. 


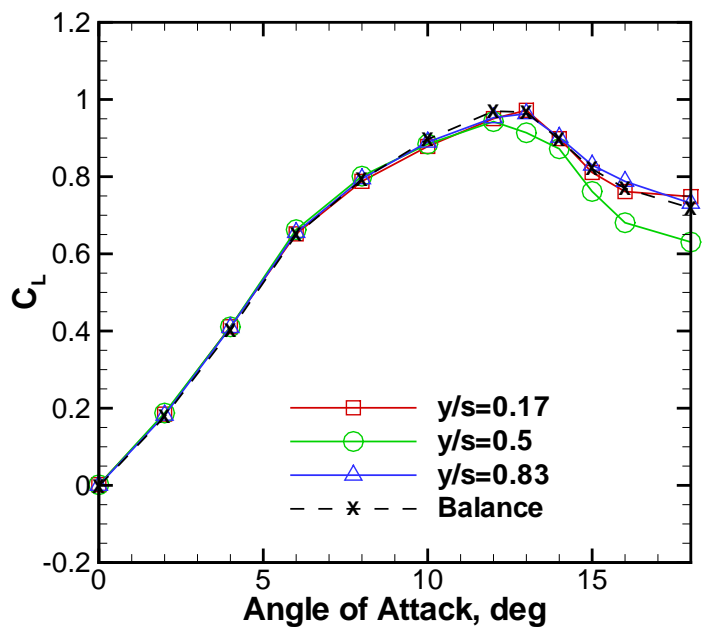

(a) $C_{L}$

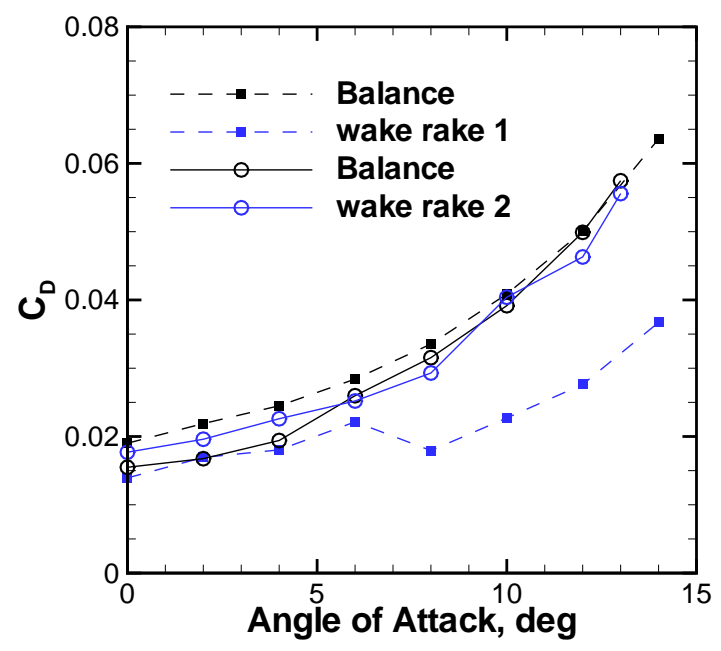

(c) $C_{D}$ from Balance and Wake Survey

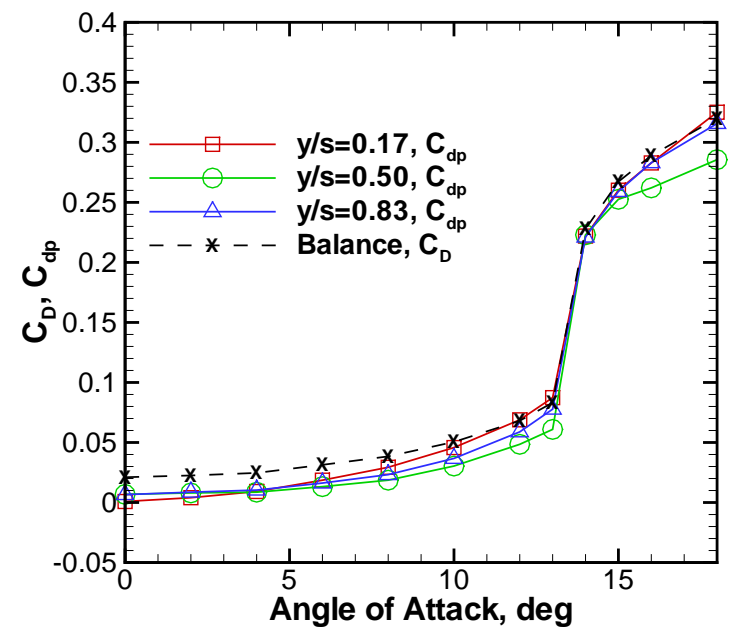

(b) $C_{D}$

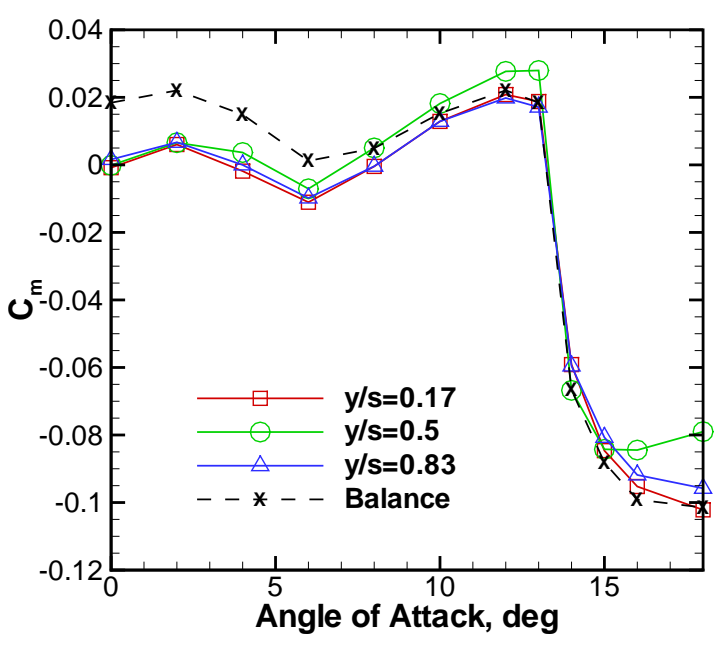

(d) $C_{m}$

Figure 2. Force and moment results for the baseline flowfield with $\delta_{f}=0^{\circ}$. $R e_{c}=0.25 x 10^{6}$. 


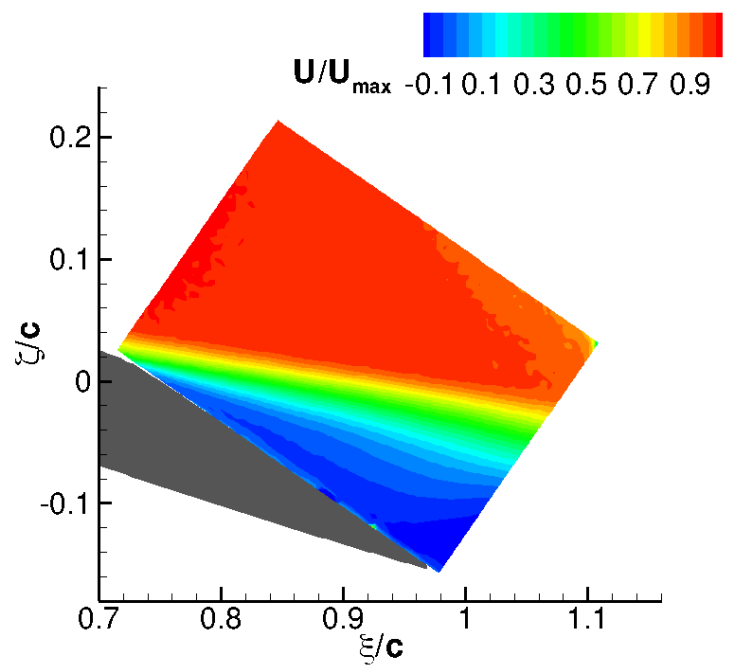

(a) $\delta_{f}=20^{\circ}$.

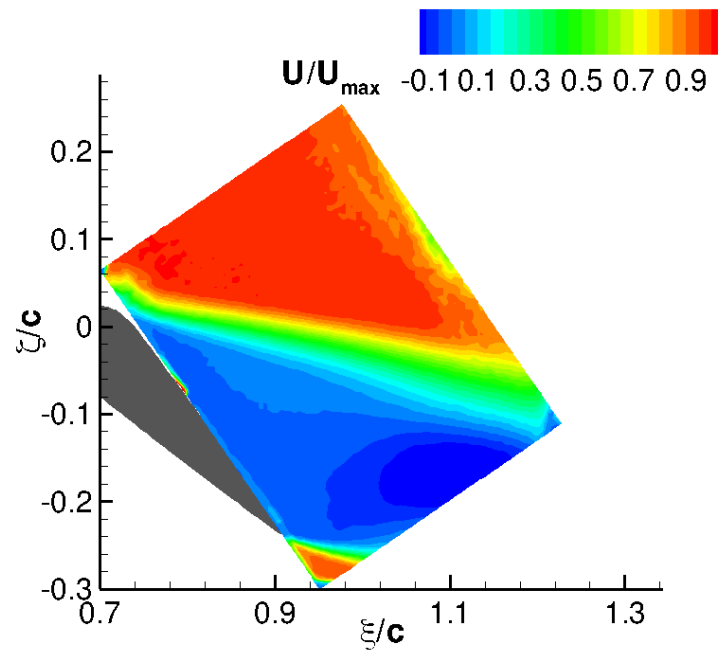

(b) $\delta_{f}=40^{\circ}$.

Figure 3. Normalized velocity contours comparing $\delta_{f}=20^{\circ}$ and $\delta_{f}=40^{\circ}$ flowfields. $\mathbf{R e}_{c}=\mathbf{0 . 2 5}^{2} \mathbf{1 0} 0^{6}, \alpha=6^{\circ}$.

\section{B. Controlled Trailing Edge Flap Flowfield}

In this section, we present trailing edge flap separation control results for $\delta_{f}=20^{\circ}$ and $\delta_{f}=40^{\circ}$. The two flap deflections, $\delta_{f}=20^{\circ}$ and $\delta_{f}=40^{\circ}$, studied were chosen because they represent a moderate and high flap deflection angle and there is existing data in the literature for comparison. With the exception of the lift as a function of angle of attack data presented, the model is at an angle of attack of 6 degrees. This angle of attack is a more difficult case for separation control because the larger adverse pressure gradient and lower momentum flow require larger momentum additions for flow reattachment than angles of attack near $\alpha=0^{\circ}$, as noted in reference 15 .

The lift increments obtained when applying $F^{+}=0.71$ control with $\alpha=6^{\circ}$ and $R e_{c}=0.25 \times 10^{6}$ are shown in figure 4 for the two flap deflection angles. Integrated lift data at three spanwise locations are compared with balance data. The lift increment, obtained when excitation is introduced at the flap shoulder, decreases as $\mathrm{y} / \mathrm{s}$ increases. The spanwise variations in lift increment observed in figures 4(a) and 4(b) agree with the actuator bench-top calibration data that showed excitation slot velocity decreases as $\mathrm{y} / \mathrm{s}$ increases. The data also indicate that $C_{L}$ increases as the excitation amplitude, $\mathrm{C}_{\mu}$, increases. The comparison of the balance lift data with the integrated lift data reveals that the balance data agree well with the $\mathrm{y} / \mathrm{s}=0.5$ integral data and both appear to represent the average lift of the model. If one then uses the balance data for comparing performance improvements, to obtain a lift increment of 0.4 , requires $\mathrm{C}_{\mu}=1.3 \%$ for $\delta_{f}=20^{\circ}$ and $\mathrm{C}_{\mu}=2.1 \%$ for $\delta_{f}=40^{\circ}$. Drag results presented in figures 4(c) and 4(d) are not as straightforward to interpret. Control increases $C_{d p}$ at $\mathrm{y} / \mathrm{s}=0.17$ whereas $C_{d p}$ at $\mathrm{y} / \mathrm{s}=0.5$ and $\mathrm{y} / \mathrm{s}=0.83$ remain below the baseline value. The $C_{d p}$ data shown in figure $4(\mathrm{c})$, reaches a minimum below $C_{\mu} \approx 0.5 \%$ at $\mathrm{y} / \mathrm{s}=0.50$ and $\mathrm{y} / \mathrm{s}=0.83$, thereafter $C_{d p}$ increases. The balance results indicate that total drag decreases as excitation amplitude, $C_{\mu}$ increases. Similar trends in $C_{D}$ and $C_{d p}$ were observed for other excitation frequencies. The variation of lift with angle of attack is shown in figures 5 comparing the baseline and controlled flowfields. The lift increment between baseline and controlled flowfields indicates, as expected, that the application of control results in a more effective flap.

The $C_{p}$ distributions for $\delta_{f}=20^{\circ}$ presented in figure $4(\mathrm{e})$ and $\delta_{f}=40^{\circ}$ presented in figure $4(\mathrm{f})$ obtained along the centerline $(\mathrm{y} / \mathrm{s}=0.5)$ of the model indicate that the zero mass flux excitation introduced at the flap shoulder increases circulation upstream of the excitation slot and delays separation on the trailing edge flap. The flow over the baseline flap is separated over the entire flap for both flap deflection angles, evidenced by the plateau in pressures on the flap. As noted in other active separation control studies ${ }^{2}$ using a NACA 0015, control at the flap shoulder results in a more positive trailing edge pressure making the trailing edge pressure an indicator of the effectiveness of the applied excitation for controlling flap separation. Separation 
is almost completely eliminated for $\delta_{f}=20^{\circ}$ with $\mathrm{C}_{\mu}=1.50 \%$. The trailing edge $C_{p}$ values for $\delta_{f}=40^{\circ}$ in figure 4 (f) indicate that the $\mathrm{C}_{\mu}=2.54 \%$ excitation does not delay separation significantly, but there is some pressure recovery (pressure increases as $\mathrm{x} / \mathrm{c}$ increases for $x / c>0.75$ ) for this excitation level compared to the baseline and $\mathrm{C}_{\mu}=0.92 \% C_{p}$ distributions.

\section{PIV Results}

In the following sections, phase-locked vorticity data from PIV measurements will be presented to determine how the excitation introduced at the flap shoulder interacts with the separating shear layer for the two flap deflections. Results will be presented for the $\delta_{f}=20^{\circ}$ airfoil followed by the $\delta_{f}=40^{\circ}$ airfoil.

\section{TE AFC with $\delta_{f}=20^{\circ}$}

A reduced frequency in the range of $0.3<F^{+}<4.0^{3}$ is considered the most effective frequency for controlling separation on a 2D airfoil. Data acquired but not presented indicated an insensitivity to frequency within the frequency range of the actuator when considering lift increment. Drag was observed to decrease with increasing frequency as noted in reference 16 . The $\delta_{f}=20^{\circ} \mathrm{PIV}$ data was obtained at $F^{+}=0.71$ corresponding to an excitation frequency of $100 \mathrm{~Hz}$ due to the insensitivity of $\Delta C_{L}$ to frequency and the improved spanwise uniformity at lower actuation frequencies. The $C_{p}$ distributions at $\mathrm{y} / \mathrm{s}=0.5$ presented in figure $4(\mathrm{e})$ indicate that as $C_{\mu}$ increases the extent of separated flow over the trailing edge flap surface is reduced. Mean vorticity $\left(\Omega * c / U_{\infty}\right)$ contours for the baseline flowfield are presented in figure 6 and the negative vorticity data indicate the mean location of the separated shear layer relative to the flap surface.

Phase-locked vorticity contours are presented in figure 7 for the $C_{\mu}$ levels of figure $4(\mathrm{e})$. In each phase of the excitation cycle when using $C_{\mu}=0.42 \%$ (Fig. 7(a), 7(d), 7(g), and $7(\mathrm{j})$ ), distinct vortex structures are observed in the flowfield. These coherent structures introduced into the flowfield are incapable of bringing the shear layer to the flap surface. The vorticity contours for the $C_{\mu}=0.89 \%$ excitation, shown in figures $7(\mathrm{~b}), 7(\mathrm{e})$, $7(\mathrm{~h})$, and $7(\mathrm{k})$, indicate that this level of excitation is capable of deflecting the shear layer towards the flap surface. In comparison to the $C_{\mu}=0.42 \%$ data of figures $7(\mathrm{a}), 7(\mathrm{a}), 7(\mathrm{~g})$, and $7(\mathrm{j})$, the additional momentum results in a significant improvement in airfoil performance. The $C_{\mu}=1.58 \% \mathrm{ex}-$ citation data of figures $7(\mathrm{c}), 7(\mathrm{f}), 7(\mathrm{i})$, and $7(\mathrm{l})$ indicate that the shear layer is at the flap surface over most of the flap chord. There is a vortex that begins to form at $\phi=270^{\circ}(7(\mathrm{l}))$, rolls up at $\phi=0^{\circ}(7(\mathrm{c}))$, has traveled almost out of the field of view of the data at $\phi=90^{\circ}(7(\mathrm{f}))$, and is out of the field of view at $\phi=180^{\circ}$ (7(i)). A question that comes to mind when looking at the data in terms of a more efficient approach to separation control, is whether locating a second actuator on the flap near $\xi / c=0.8$ would reduce the total momentum requirement for complete flow reattachment to the flap.

\section{TE AFC with $\delta_{f}=40^{\circ}$}

The $\delta_{f}=20^{\circ}$ vorticity data of figure 7 illustrated how the $F^{+}=0.71$ periodic excitation introduced into the separated shear layer interacted with the separated shear layer to delay the average separation location on the upper surface of the flap. Similar data will be presented in this section for the airfoil when $\delta_{f}=40^{\circ}$. The airfoil angle of attack is fixed at $\alpha=6^{\circ}$. One of the most noticeable differences between the $\delta_{f}=20^{\circ}$ and the $\delta_{f}=40^{\circ}$ baseline flowfields is the presence of a dominant frequency in the voltage signal of practically every dynamic pressure transducer on the model for the $\delta_{f}=40^{\circ}$ case. For the freestream velocity of this test, $\mathrm{U} \approx 13 \mathrm{~m} / \mathrm{s}$, the frequency is $\mathrm{f}=47 \mathrm{~Hz}$ as shown in figure 8. Since the actuators used for control were calibrated between the range of $50 \mathrm{~Hz}$ and $400 \mathrm{~Hz}$, we excited the flow at a frequency of $50 \mathrm{~Hz}$ corresponding to $F^{+}=0.36$ to determine the response of the flowfield to this $F^{+}$as well as at $F^{+}=0.71$. The variations of $C_{L}, C_{D}$ and $C_{d p}$ with $C_{\mu}$ are presented in figure $9(\mathrm{a})$ and figure 9(b). The momentum requirements for a given lift increment are reduced compared to the $\delta_{f}=40^{\circ}, F^{+}=0.71$ excitation of figure 4(b). 


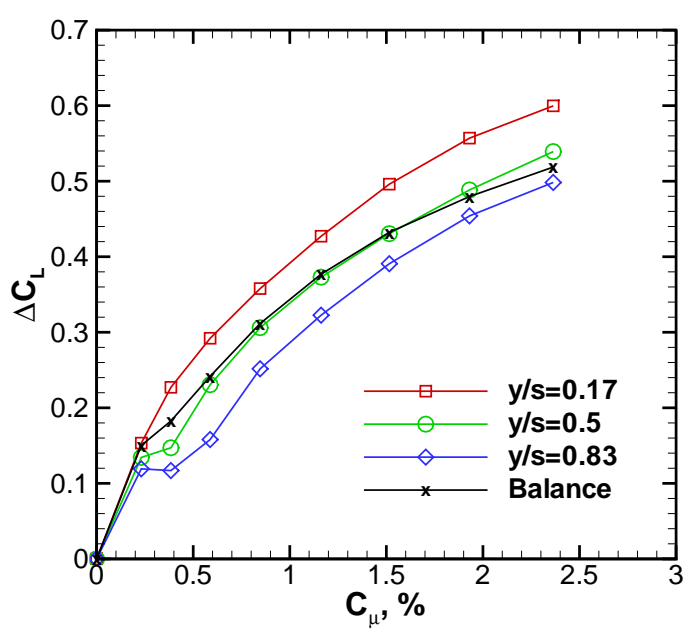

(a) $\delta_{f}=20^{\circ}, \Delta C_{L}$.

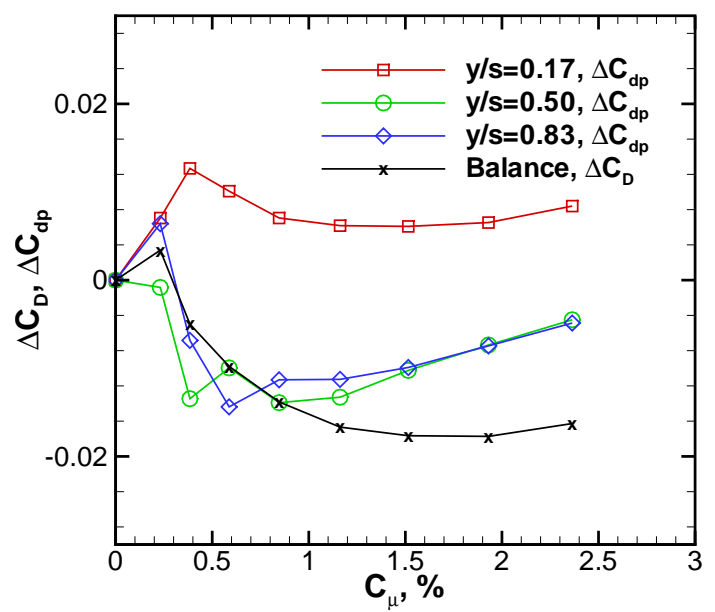

(c) $\delta_{f}=20^{\circ}, \mathrm{F}^{+}=0.71, \Delta C_{D}, d p$.

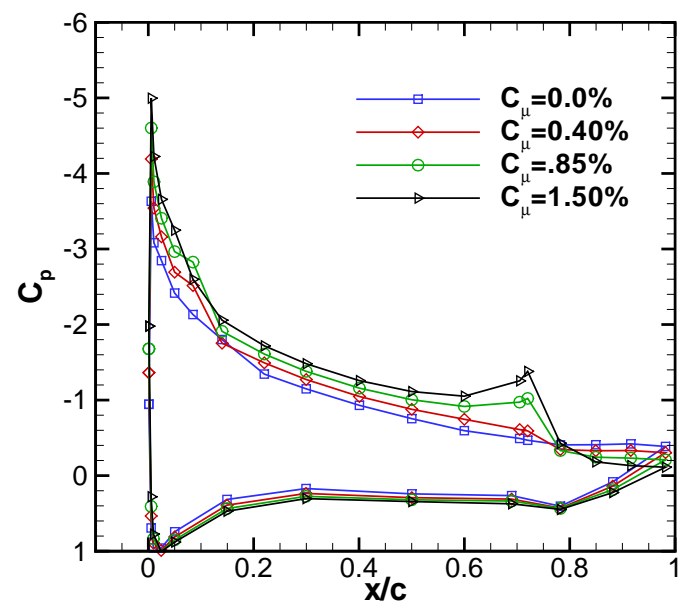

(e) $\delta_{f}=20^{\circ}, C_{p}$.

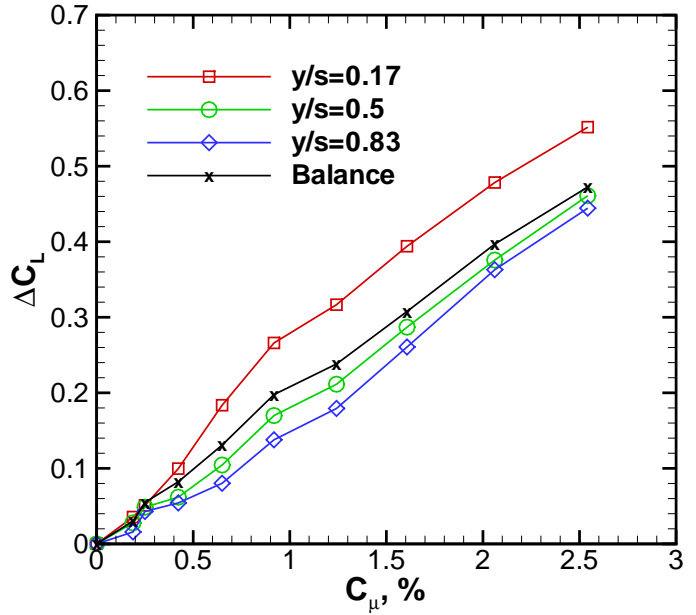

(b) $\delta_{f}=40^{\circ}, \Delta C_{L}$

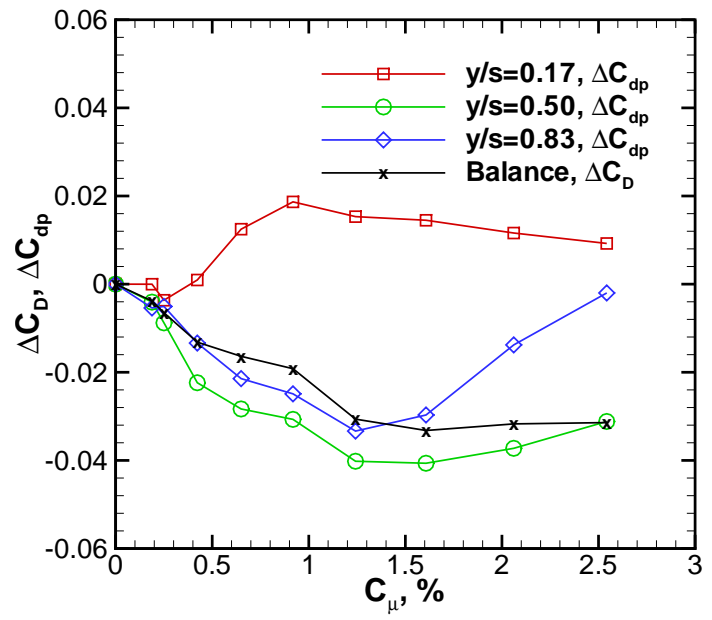

(d) $\delta_{f}=40^{\circ}, \Delta C_{D}, d p$

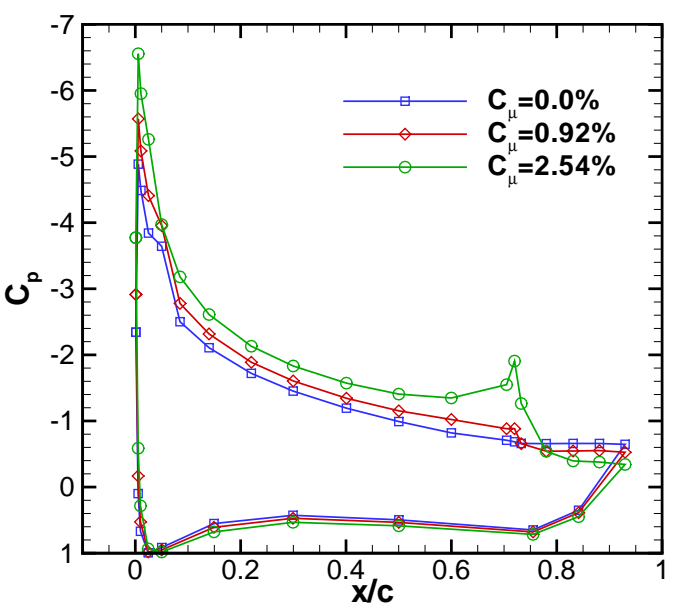

(f) $\delta_{f}=40^{\circ}, C_{p}$.

Figure 4. Lift increment due to trailing edge flap control. $\operatorname{Re}_{c}=0.25 \times 10^{6}, \mathrm{~F}^{+}=\mathbf{0 . 7 1}$. 10 of 18 


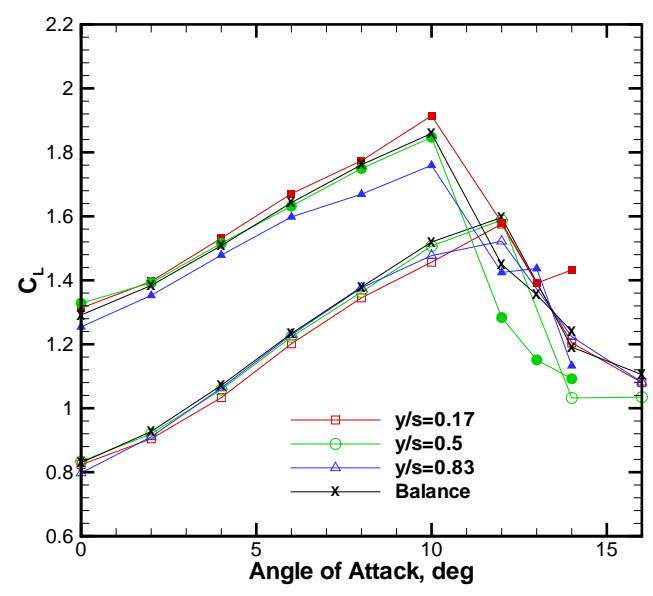

(a) $\delta_{f}=20^{\circ}, \mathrm{C}_{\mu}=1.35 \%$.

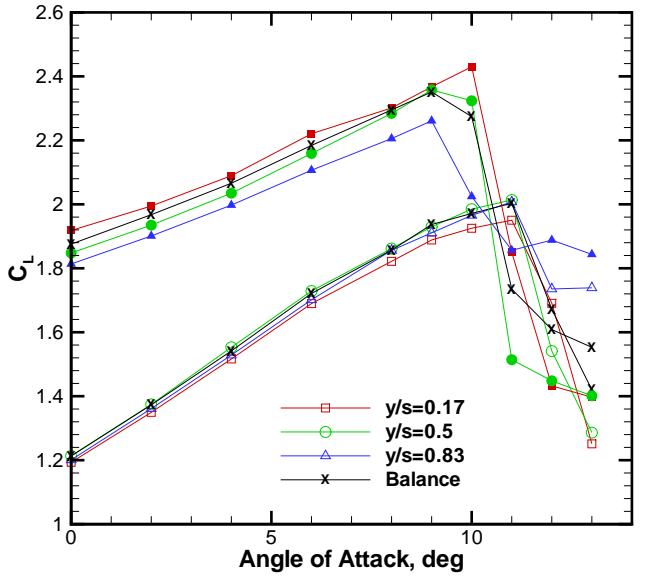

(b) $\delta_{f}=40^{\circ}, \mathrm{C}_{\mu}=2.5 \%$.

Figure 5. Lift data comparison baseline and controlled flowfields. $\mathbf{R e}_{c}=0.25 \times 10^{6}, F^{+}=0.71$.

The excitation causes an increase in drag for most $C_{\mu}$ levels. It is only between $C_{\mu}=0.2 \%$ and $0.4 \%$ that a reduction in $C_{D}$ and $C_{d p}$, outboard of y/s=0.17, occur. In figure $9(\mathrm{c})$ balance data are presented for other $F^{+}$ values tested and small variations in lift are noted with changes in $F^{+}$between $F^{+}=0.64$ and $F^{+}=1.43$. The effectiveness of the lower $F^{+}$excitation and the insensitivity to frequency between $F^{+}=0.64$ and $F^{+}=1.43$ are in agreement with the observations of Ref. 17. The $C_{p}$ distributions of figure $9(\mathrm{~d})$ show that the $F^{+}=0.36$ excitation is more effective at increasing circulation than the $F^{+}=0.71$ excitation. The pressure recovery generated by the $F^{+}=0.71, C_{\mu}=2.54 \%$ excitation, included in figure 9 (d) for comparison, indicates that $F^{+}=0.71$ is better at controlling separation on the flap of the model.

Phase-locked, normalized vorticity contours obtained from 2D PIV measurements are presented in figure 10 for the airfoil with $\delta_{f}=40^{\circ}$. Two $F^{+}$values are compared using two $C_{\mu}$ levels for the $F^{+}=0.71$ excitation. The vorticity contours for the flowfield controlled with the $F^{+}=0.36$ excitation are provided in figures $10(\mathrm{a}), 10(\mathrm{~d}), 10(\mathrm{~g})$, and $10(\mathrm{j})$ with $C_{\mu}=0.70 \%$. The excitation causes the negative vorticity of the upper surface of the flap to roll up into discrete structures that are shed into the wake. The excitation also moves the location of the positive vortex that is shed from the lower surface of the flap upstream so that it is closer to the trailing edge of the model (Fig. 10(d)). In comparison to lower amplitude data (not included), the negative vortex is also closer to the flap surface. Separation is delayed when using $C_{\mu}=0.70 \%$ at $F^{+}=0.36$. However, the increment in lift obtained with the $F^{+}=0.36$ excitation compared to the $F^{+}=0.71$ excitation, is due to changes in circulation caused by altering the vortex shedding pattern.

The $F^{+}=0.71$ phase-locked, normalized vorticity data are pre-

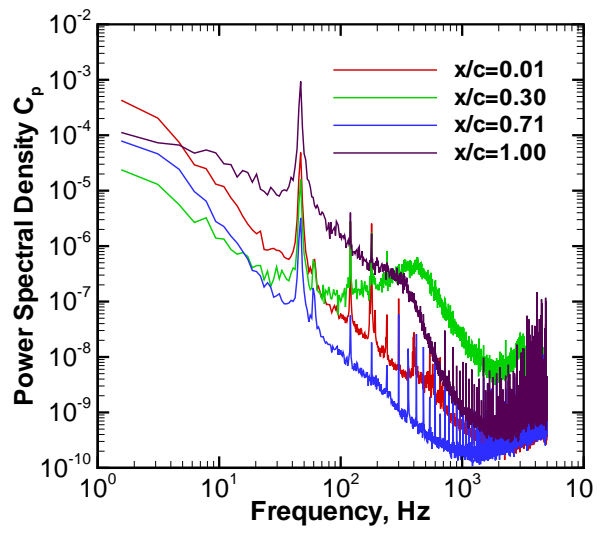

Figure 8. Spectra of $C_{p}$ from unsteady pressure transducers at $\mathrm{y} / \mathrm{s}=\mathbf{0 . 4 9 6} . \delta_{f}=20^{\circ}$.

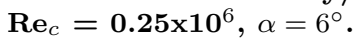
sented in figures $10(\mathrm{~b}), 10(\mathrm{e}), 10(\mathrm{~h})$ and $10(\mathrm{k})$ for $C_{\mu}=0.92 \%$.

The excitation at this level, generated only weak vortex structures in the flowfield. The mean position of the shear layer above the flap does not appear to be altered by the excitation. Additional data near the excitation slot, not acquired for this test, are required to determine how much the excitation delays separation. When the $C_{\mu}$ level of the excitation is increased to $2.54 \%$ (figures 10(c), 10(f), 10(i) and 10(l)), stronger coherent structures are formed. The vortex structures, formed by the interaction of the excitation with the 


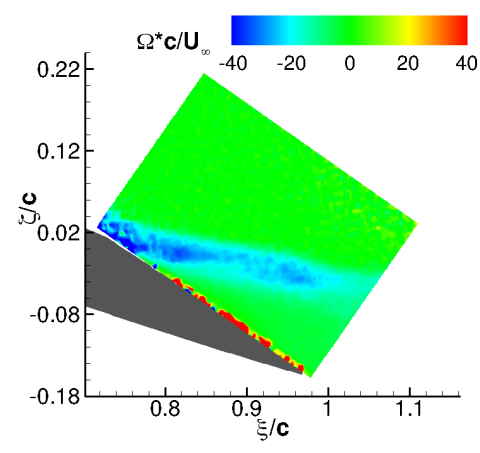

(a) $\phi=0^{\circ}, C_{\mu}=0.42 \%$.

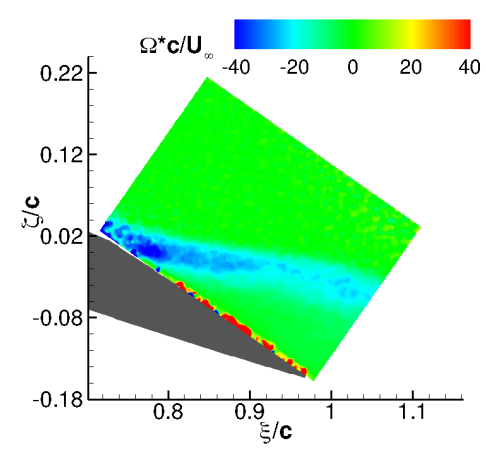

(d) $\phi=90^{\circ}, C_{\mu}=0.42 \%$.

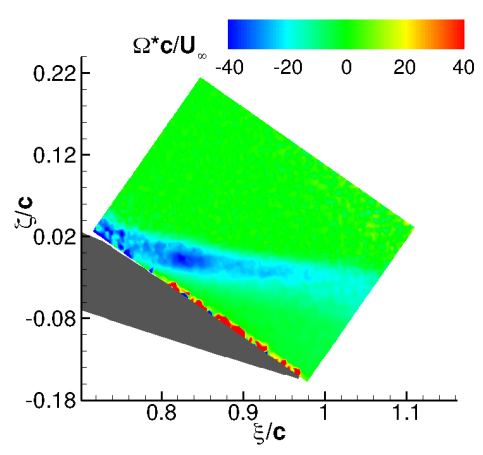

(g) $\phi=180^{\circ}, C_{\mu}=0.42 \%$.

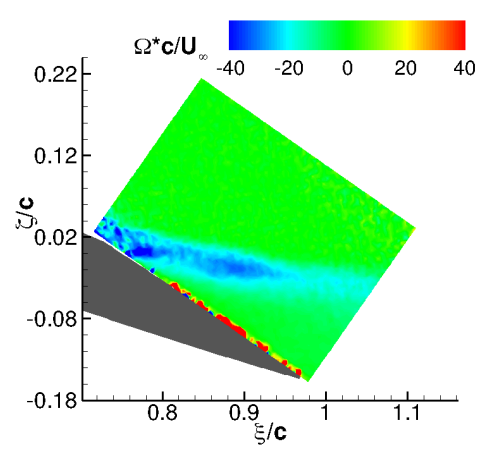

(j) $\phi=270^{\circ}, C_{\mu}=0.42 \%$.

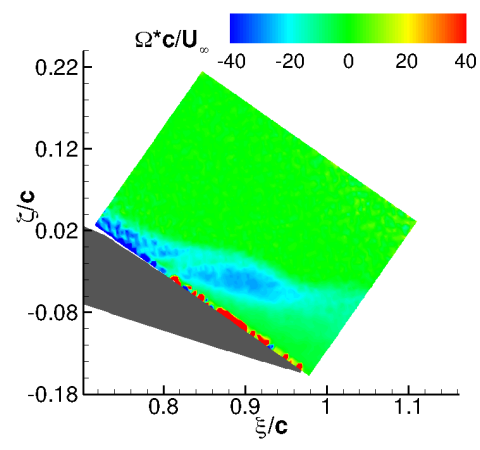

(b) $\phi=0^{\circ}, C_{\mu}=0.89 \%$.

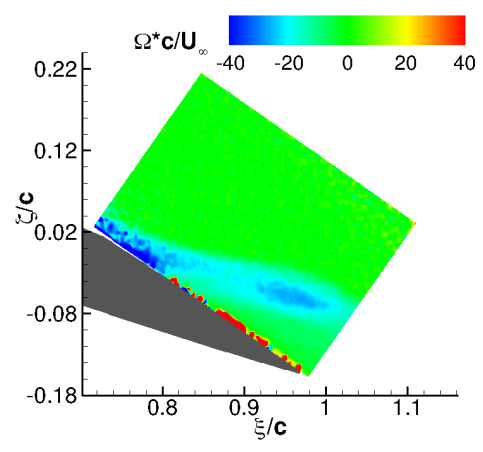

(e) $\phi=90^{\circ}, C_{\mu}=0.89 \%$.

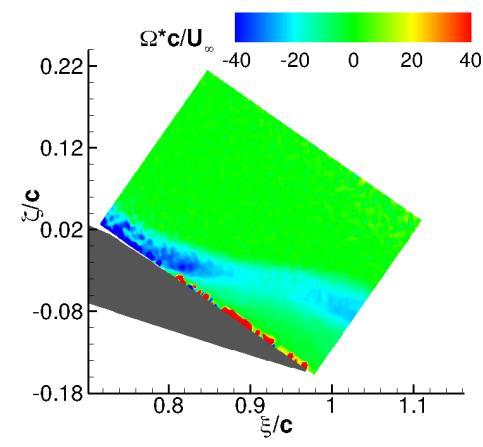

(h) $\phi=180^{\circ}, C_{\mu}=0.89 \%$.

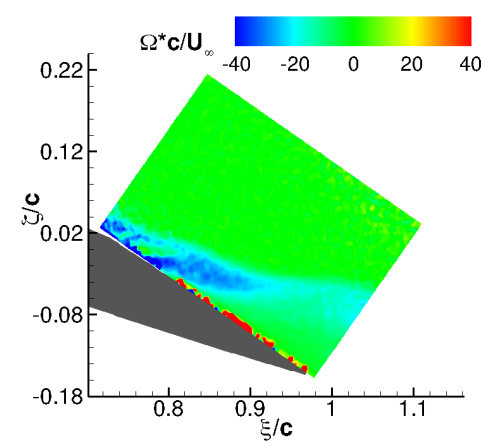

(k) $\phi=270^{\circ}, C_{\mu}=0.89 \%$.

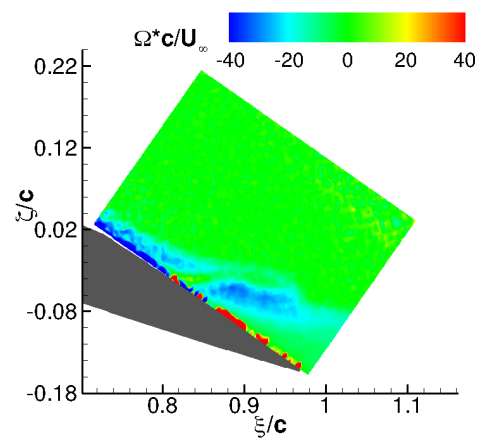

(c) $\phi=0^{\circ}, C_{\mu}=1.58 \%$.

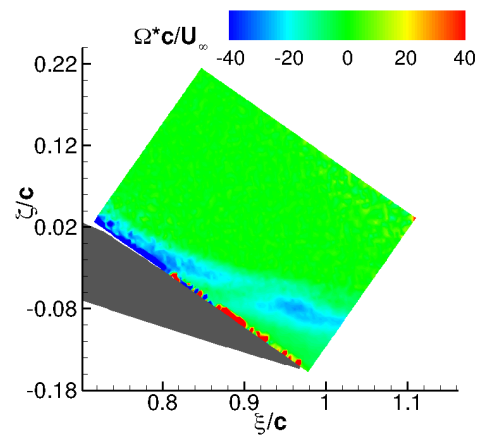

(f) $\phi=90^{\circ}, C_{\mu}=1.58 \%$.

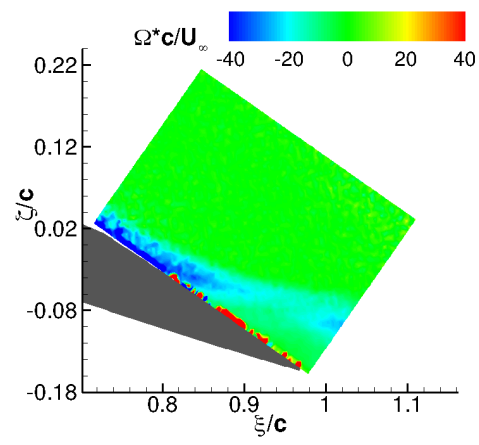

(i) $\phi=180^{\circ}, C_{\mu}=1.58 \%$.

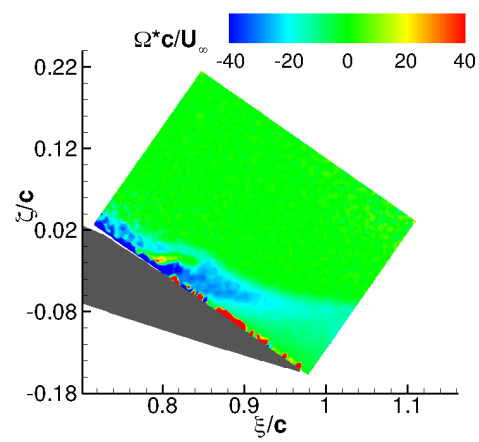

(l) $\phi=270^{\circ}, C_{\mu}=1.58 \%$.

Figure 7. Phase-locked, normalized vorticity $\left(\Omega * c / U_{\infty}\right)$ contours from PIV measurements with $\delta_{f}=20^{\circ} . \mathbf{R e}_{c}$ $=0.25 \times 10^{6}, \alpha=6^{\circ}, F^{+}=\mathbf{0 . 7 1}$. 


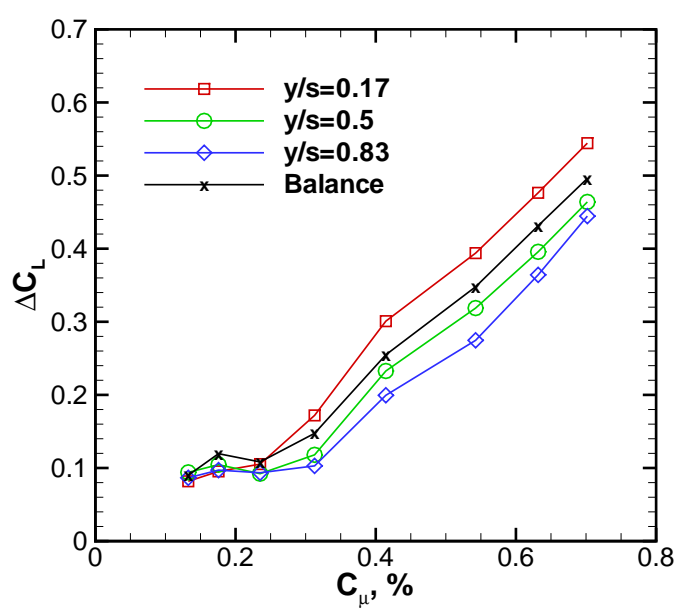

(a) $C_{\mu}$ effect on lift for $F^{+}=0.36$.

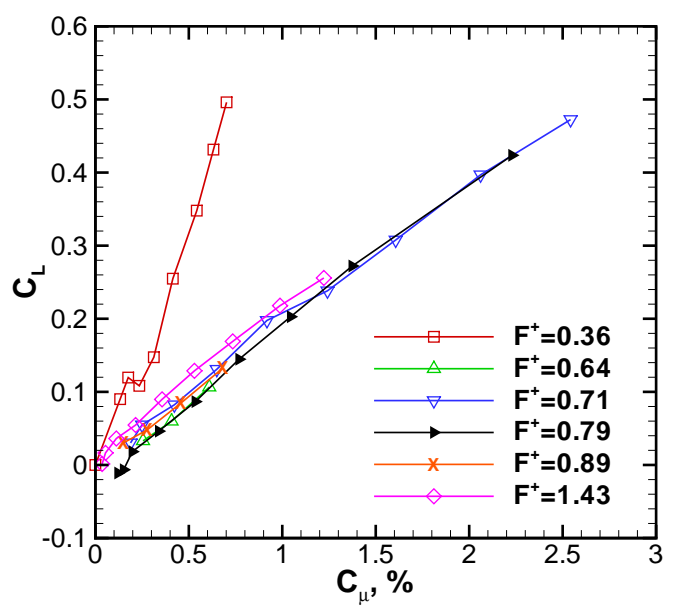

(c) $C_{\mu}$ effect on lift, $F^{+}$varied.

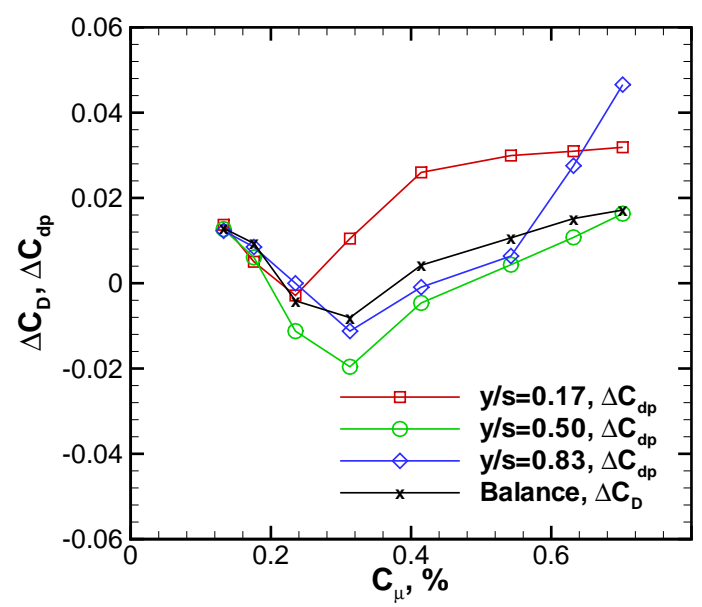

(b) $C_{\mu}$ effect on drag for $F^{+}=0.36$.

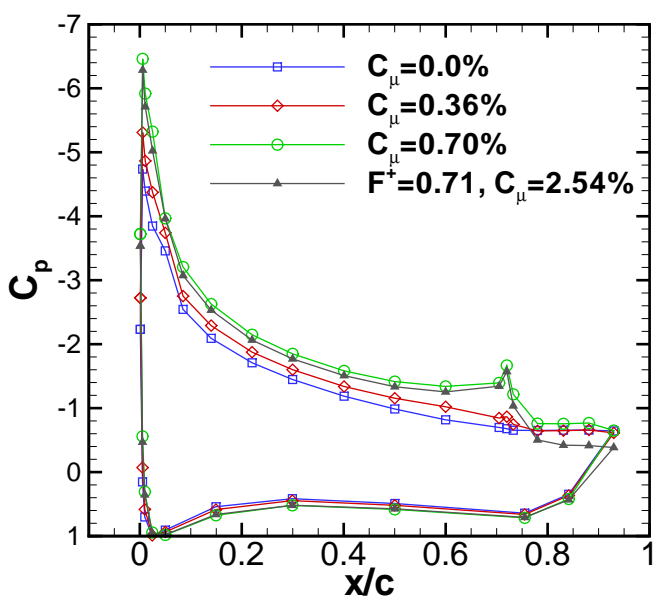

(d) $C_{p}$ distributions for $F^{+}=0.36$.

Figure 9. Lift increment, lift, and $C_{p}$ results for the $\delta_{f}=40^{\circ}$ flowfield. $\mathbf{R e}_{c}=\mathbf{0 . 2 5 \times 1 0 ^ { 6 }}$. 
separating shear layer, do not deflect the shear layer towards the surface of the flap as effectively as the vortex structures present in the $\delta_{f}=20^{\circ}, C_{\mu}=1.58 \%$ excited flowfield (figures $7(\mathrm{c}), 7(\mathrm{f}), 7(\mathrm{i})$, and $7(\mathrm{l})$ ).

The differences in the response of the $\delta_{f}=40^{\circ}$ flowfield to the two slightly different frequencies and thus excitation wavelengths suggest that excitation using a modulated waveform might be beneficial. Modulating the excitation has been proven to reduce the momentum requirements for controlling separation in cases where the excitation frequency being modulated is outside of the range of $0.5<F^{+}<2.0{ }^{5}$ Burst modulation will be used in the next section to examine the response of the flowfield to periodic excitation. An excitation comprised of periodic excitation with either steady suction ${ }^{18}$ or blowing ${ }^{1}$ as suggested in reference 7 might be a more efficient method of controlling TE flap separation when $\delta_{f}=40^{\circ}$ and will be explored at a later date.

\section{Transient Process}

In this section we briefly examine the transient process associated with attaching and separating the flow on the flap of the model. Darabi and Wygnanski ${ }^{19,20}$ have performed a detailed study of both the separation and reattachment processes. They list maneuvering by separation control and closed-loop separation control as reasons for examining the transients of both separation and reattachment. We are studying the transients in preparation for future closed-loop control research. The method used to introduce the perturbation is the method that Greenblatt ${ }^{9}$ used during the semi-span test of this model. The airfoil with $\delta_{f}=20^{\circ}$ is at $\alpha=6^{\circ}$ and the sinusoidal waveform that has been used throughout the investigation is modulated $(\mathrm{M})$ with a lower frequency pulse $\left(f_{M}\right)$ having a duty cycle of $50 \%$. This method of control, depicted in figure 11 along with the trailing edge actuator cavity pressure, will be referred to as burst mode (BM). The $C_{\mu}=1.58 \%$ excitation of the $F^{+}=0.71$ excitation does not fully attach the separated flow on the suction surface of the flap as shown in figure 12. However, the ability of the actuator operating in BM to return the trailing edge $C_{p}$ to the value, $C_{p}=-0.1$, obtained when using a continuous sine wave (figure 12), will be considered flow attachment. Data from the trailing edge dynamic pressure transducer installed on the model at $\mathrm{y} / \mathrm{s}=0.5$ are presented in figure 13(a). Lift data from the balance are also included in figure 13(b) for comparison. The balance data are included recognizing that the frequency response of the balance may not be sufficient to capture all of the details of the response to the input. It should also be noted that the dynamic pressure value used to compute $C_{p}$ and $C_{L}$ is an averaged quantity. The longer wavelength excitation produced by the $f_{M}=2.0$ $\mathrm{Hz}$ excitation is the best signal to use to determine the time required to attach the flow to the flap of the model. In figure 13, the excitation is turned on at time, $t=0 \mathrm{sec}$. At $\mathrm{t} \approx 0.024 \mathrm{~s}$, the initially separated flow is observed to obtain the same level of attachment as the continuous waveform excitation. This is also true of the flowfields controlled using the higher frequency excitations, $f_{M}=5.0 \mathrm{~Hz}$ and $25.0 \mathrm{~Hz}$ in figure $13(\mathrm{a})$. For the $f_{M}=2.0 \mathrm{~Hz}$ excitation, control is turned off at $\mathrm{t}=0.25 \mathrm{~s}$. The time for the flow to return to the initial separated state is $\mathrm{t} \approx 0.05 \mathrm{~s}$. The time to separate is the same for the $f_{M}=5.0$ excited flowfield. The $f_{M}=25.0$ $\mathrm{Hz}$ excited flowfield does not return to a fully separated state based on the trailing edge pressure data and balance lift data (Fig. 13(b)). The lift data of figure 13(b) indicate that the time required for $C_{L}$ to increase from the baseline $C_{L}$ value of 1.2 to the controlled, using a continuous sine wave, $C_{L}$ value of 1.61 is $t \approx 0.042$ s. The phase-locked normalized PIV data are provided in figure 14 to illustrate the changes in the shear layer with BM control. The phases shown have been selected to coincide with some of the transients observed in the flap TE pressure signal. The flowfield controlled using the $f_{M}=2.0 \mathrm{~Hz}\left(F_{M}^{+}=0.014\right) \mathrm{BM}$ excitation of figures 14(a), 14(d), 14(g), and 14(j), 14(m) is initially separated, attaches to the upper surface of the flap, and then separates from the flap surface as commanded by the input signal. There is an overshoot indicative of more separated flow, noticeable in the $f_{M}=2.0 \mathrm{~Hz}$ and $5.0 \mathrm{~Hz}$ data, during the separation process that starts at the arrow shown in figures 13. The overshoot (more separated flow) during the separation process is larger $\left(C_{p}\right.$ more negative) for the $f_{M}=2.0 \mathrm{~Hz}$ excitation. The vorticity data shown in figures 14(b), 14(e), $14(\mathrm{~h})$, and $14(\mathrm{k}), 14(\mathrm{n})$ for the $f_{M}=5.0 \mathrm{~Hz}\left(F_{M}^{+}=0.035\right)$ excitation case indicate that at $\mathrm{t}=0.125 \mathrm{~s}$, during the separation process, the positive vortex shed from the lower surface of the flap rolls up very near the trailing edge of the model and the shear layer has moved away from the upper surface of the flap. The $f_{M}=25.0$ $\mathrm{Hz}\left(F_{M}^{+}=0.125\right)$ excitation does not completely separate when the excitation is turned off, however there is a tendency for the flow at the TE to separate when the excitation is turned on. The vorticity contours presented in figures 14(c), 14(f), 14(i), and 14(1), 14(o) indicate at $\mathrm{t}=0.005 \mathrm{~s}$, the positive vortex shed from the lower surface is near the TE of the model and the shear layer location indicates the flow is separated. 


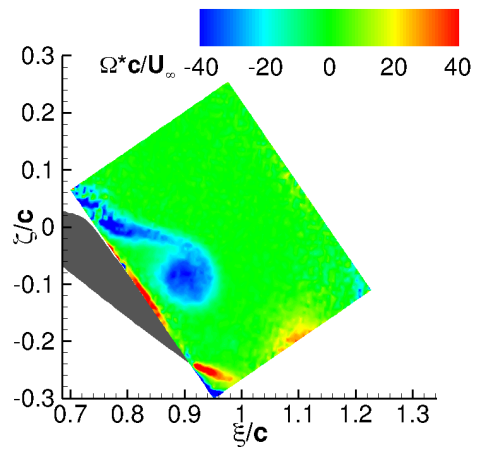

(a) $\phi=0^{\circ}, F^{+}=0.36, C_{\mu}=0.70 \%$.

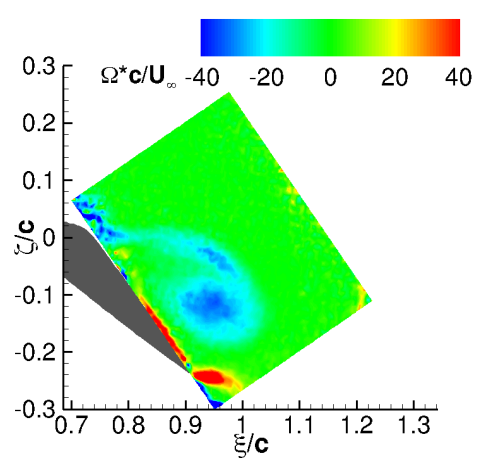

(d) $\phi=90^{\circ}, F^{+}=0.36, C_{\mu}=0.70 \%$.

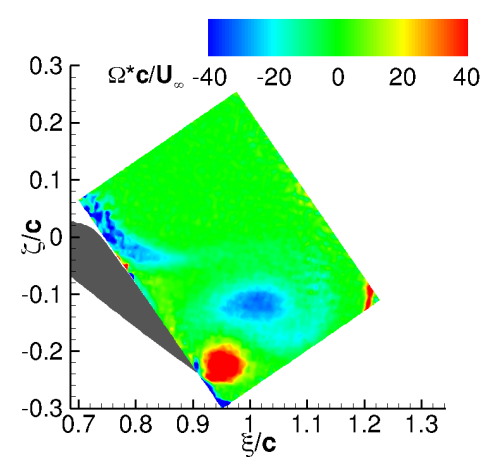

(g) $\phi=180^{\circ}, F^{+}=0.36, C_{\mu}=0.70 \%$.

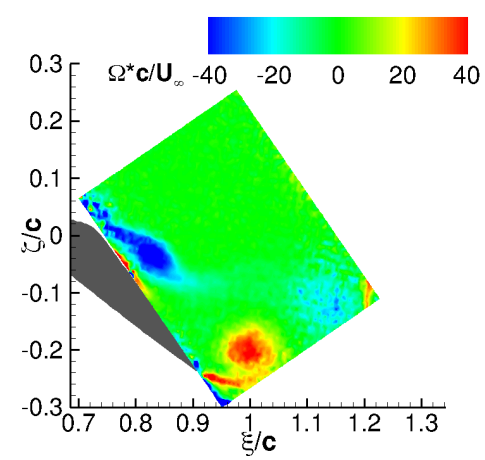

(j) $\phi=270^{\circ}, F^{+}=0.36, C_{\mu}=0.70 \%$.

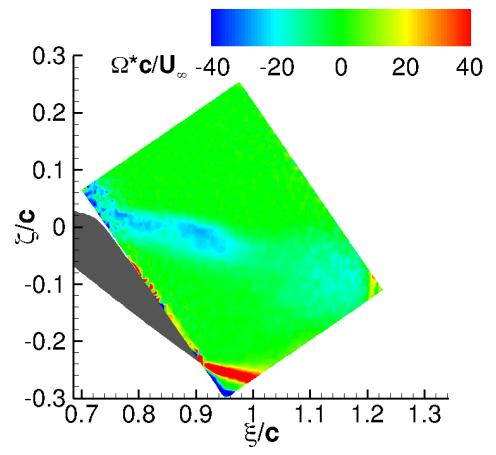

(b) $\phi=0^{\circ}, F^{+}=0.71, C_{\mu}=0.92 \%$.

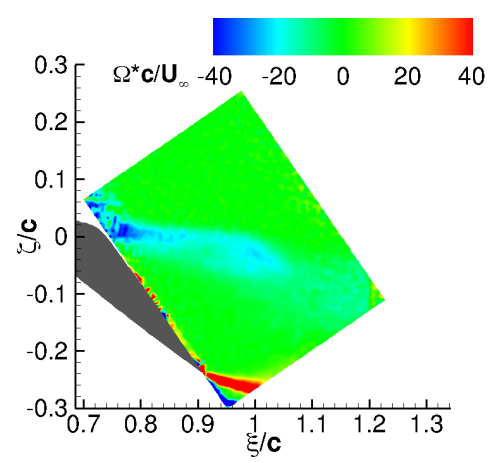

(e) $\phi=90^{\circ}, F^{+}=0.71, C_{\mu}=0.92 \%$.

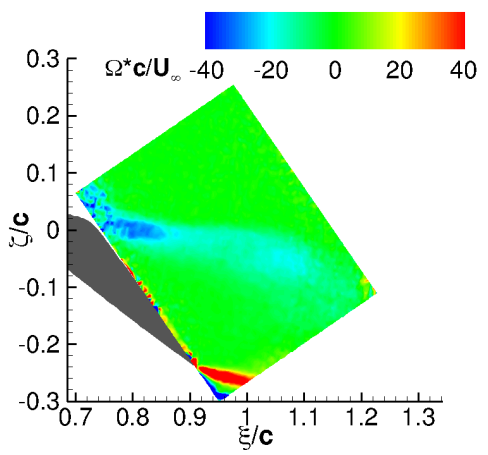

(h) $\phi=180^{\circ}, F^{+}=0.71, C_{\mu}=0.92 \%$.

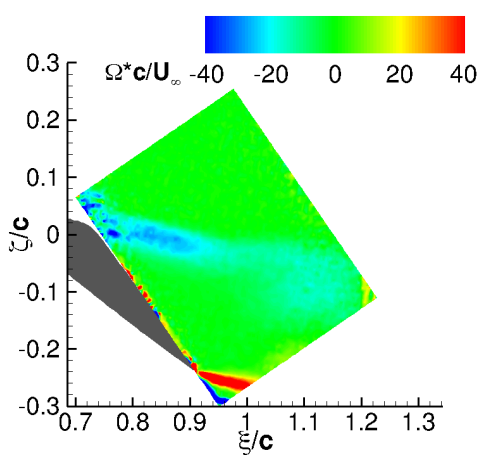

(k) $\phi=270^{\circ}, F^{+}=0.71, C_{\mu}=0.92 \%$.

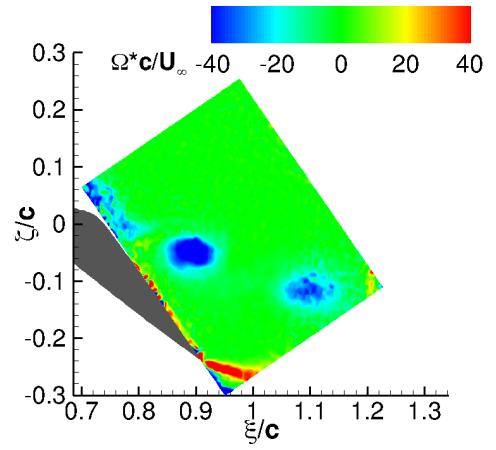

(c) $\phi=0^{\circ}, F^{+}=0.71, C_{\mu}=2.54 \%$.

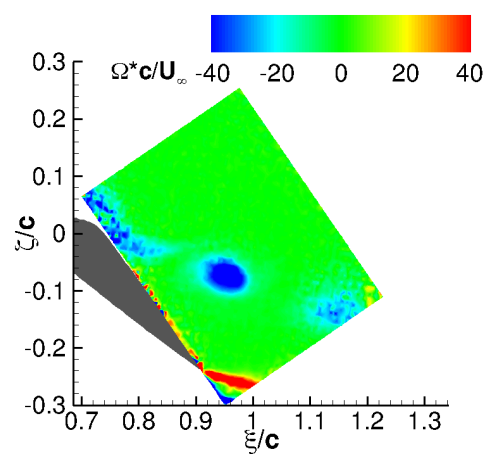

(f) $\phi=90^{\circ}, F^{+}=0.71, C_{\mu}=2.54 \%$.

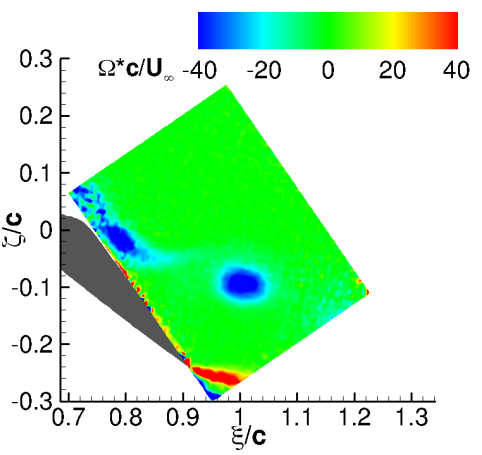

(i) $\phi=180^{\circ}, F^{+}=0.71, C_{\mu}=2.54 \%$.

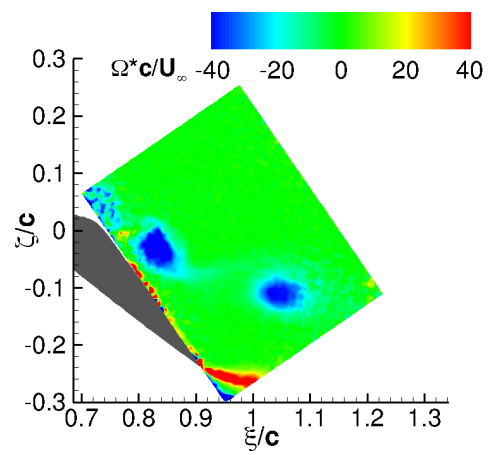

(l) $\phi=270^{\circ}, F^{+}=0.71, C_{\mu}=2.54 \%$.

Figure 10. Phase-locked, normalized vorticity contours from PIV measurements with $\delta_{f}=40^{\circ} \cdot \mathbf{R e}_{c}=\mathbf{0 . 2 5 \times 1 0 ^ { 6 }}$, $\alpha=6^{\circ}$. 


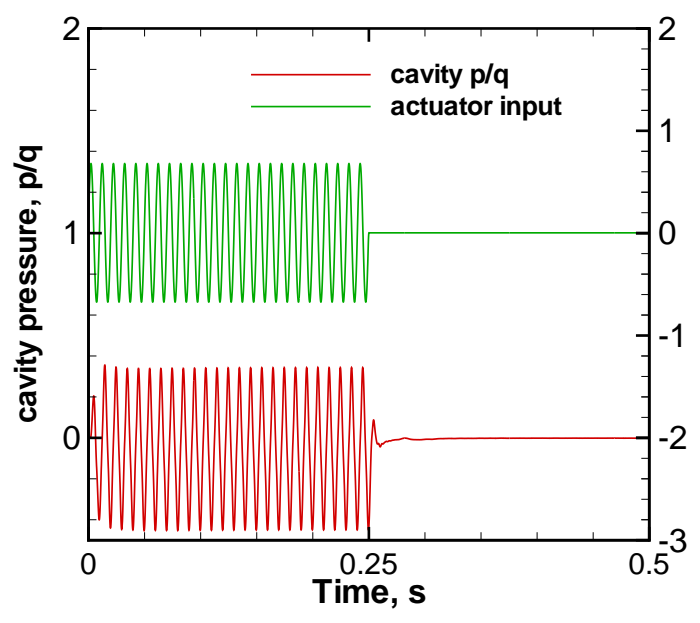

Figure 11. Actuator input signal and TE actuator cav-

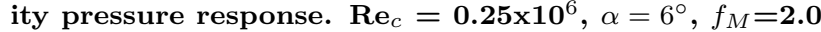
Hz.
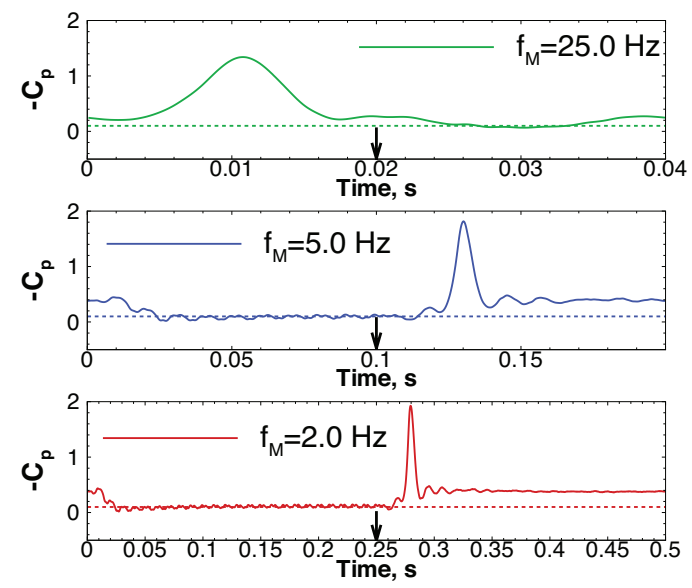

(a) TE pressure fluctuations.

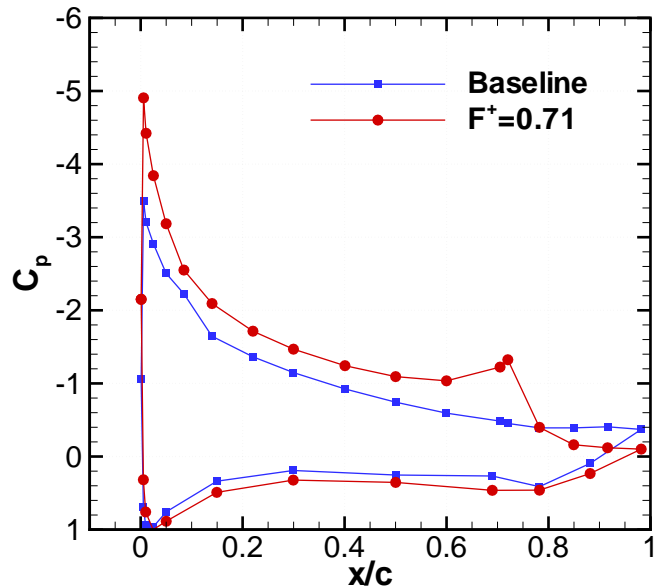

Figure 12. Effect of $F^{+}=0.71, C_{\mu}=1.46 \%$ on $C_{p}$ at $\mathrm{y} / \mathrm{s}$ $=0.5$. $\mathbf{R e}_{c}=0.25 \times 10^{6}, \alpha=6^{\circ}$.
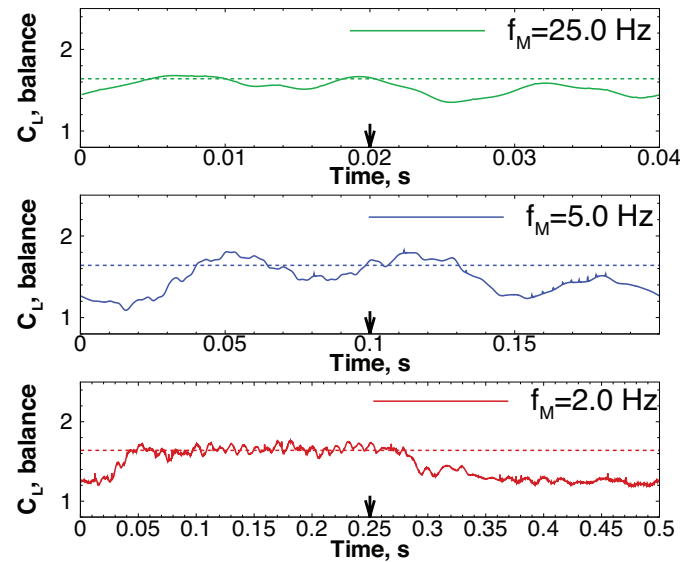

(b) Balance $C_{L}$.

Figure 13. TE $C_{p}$ and balance $C_{L}$ time histories illustrating response of $\delta_{f}=20^{\circ}$ flowfield to burst mode excitation. $\mathbf{R e}_{c}=\mathbf{0 . 2 5} \times 10^{6}, \alpha=6^{\circ}$. The arrows indicate time when excitation switched off.

\section{Summary}

As part of a series of planned wind tunnel experiments aimed at examining the flow physics of active separation control, periodic excitation was applied at the flap shoulder of a NACA 0015 airfoil and the effects of amplitude and frequency were examined. The primary objective of the experiment was to compare the results obtained using a 5-component strain gage balance to those obtained by integrating surface pressures. The research is performed in preparation for additional three-dimensional testing of the model where the balance measurements will complement the pressure measurements. We focused on controlling separation on the trailing edge flap at an angle of attack of $\alpha=6^{\circ}$. This angle was chosen because the degree of adverse pressure gradient on the flap required oscillatory momentum coefficients $\left(C_{\mu}\right)$ greater than $1 \%$ to completely attach the flow to the surface of the deflected flap. The model, previously used for three-dimensional testing, has several rows of streamwise pressures that were used to compute lift and drag and compare to the balance measurements. There was good agreement between the lift values of the two measurement techniques. Two dimensional high speed Particle Image Velocimetry (PIV) data were also presented and provided insight 


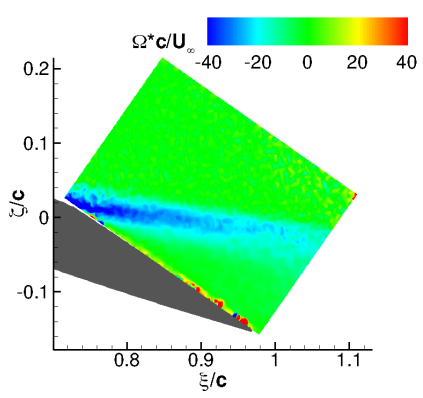

(a) t $=0.0 \mathrm{~s}, F_{M}^{+}=0.014$.

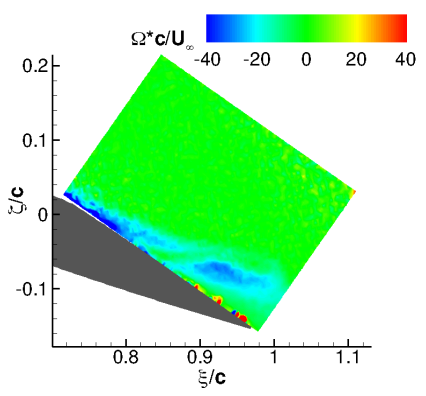

(d) $\mathrm{t}=0.063 \mathrm{~s}, F_{M}^{+}=0.014$.

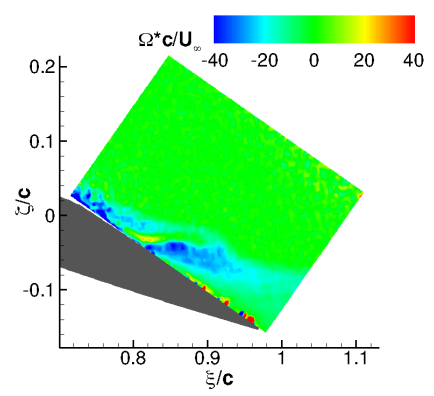

(g) t=0.250 s, $F_{M}^{+}=0.014$.

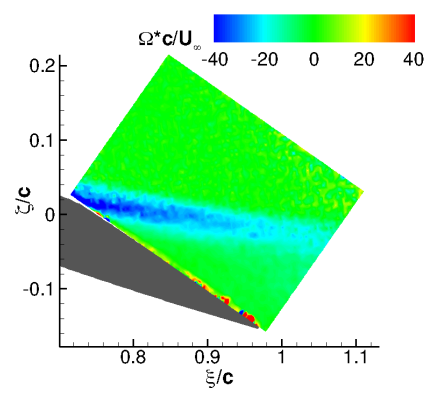

(j) t=0.31 s, $F_{M}^{+}=0.014$.

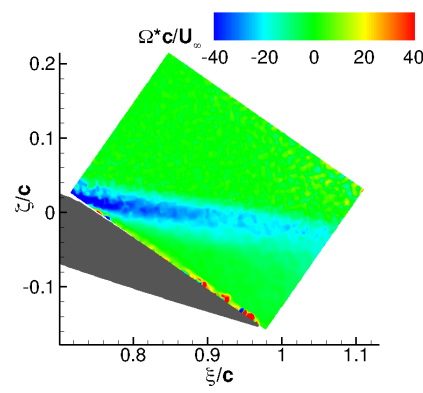

(m) t $=0.438 \mathrm{~s}, F_{M}^{+}=0.014$.

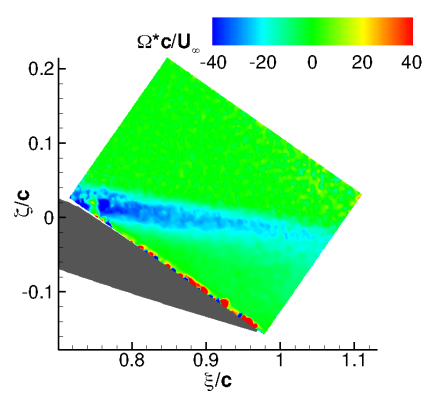

(b) t=0.0 s, $F_{M}^{+}=0.035$.

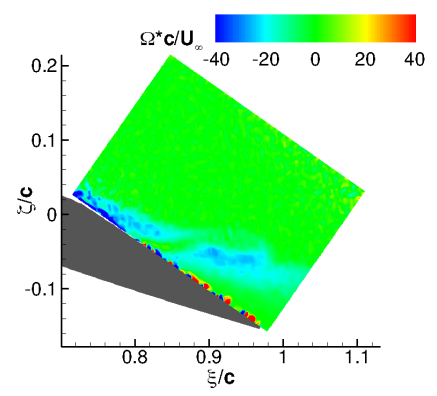

(e) $\mathrm{t}=0.025 \mathrm{~s}, F_{M}^{+}=0.035$.

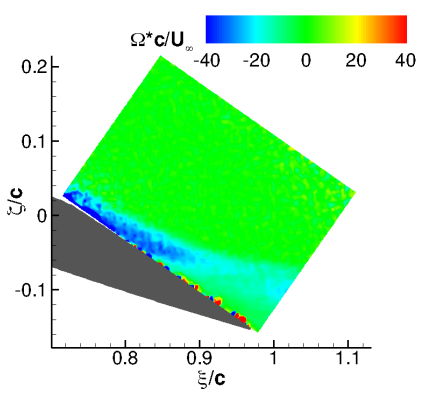

(h) t=0.10 s, $F_{M}^{+}=0.035$.

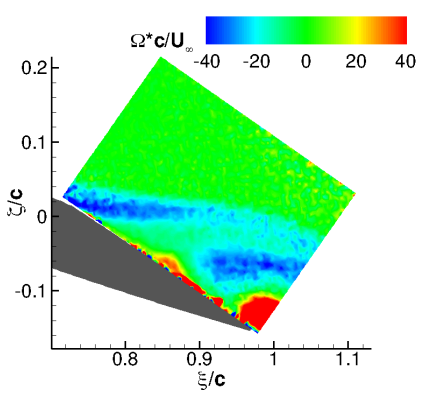

(k) t $=0.125 \mathrm{~s}, F_{M}^{+}=0.035$.

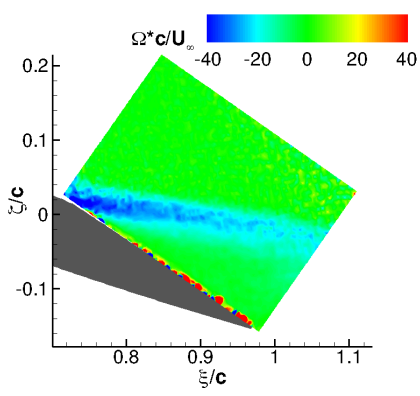

(n) $\mathrm{t}=0.175 \mathrm{~s}, F_{M}^{+}=0.035$.

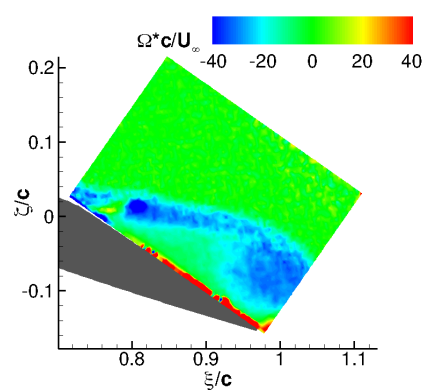

(c) $\mathrm{t}=0.0 \mathrm{~s}, F_{M}^{+}=0.18$.

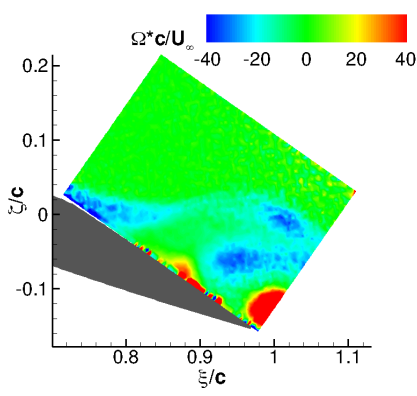

(f) $\mathrm{t}=0.005 \mathrm{~s}, F_{M}^{+}=0.18$.

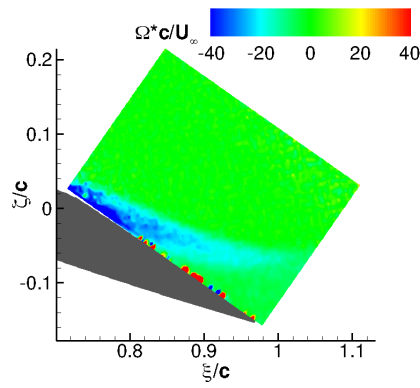

(i) $\mathrm{t}=0.02 \mathrm{~s}, F_{M}^{+}=0.18$.

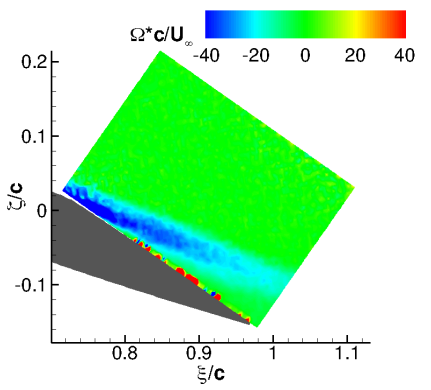

(l) $\mathrm{t}=0.025 \mathrm{~s}, F_{M}^{+}=0.18$.

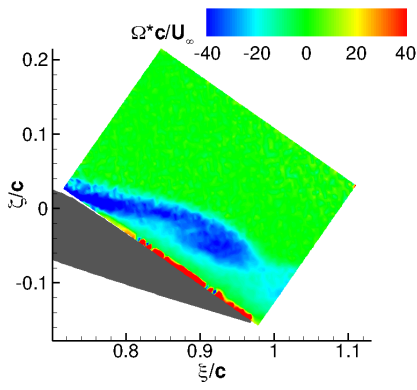

(o) $\mathrm{t}=0.035 \mathrm{~s}, F_{M}^{+}=0.18$.

Figure 14. Vorticity contours illustrating response of $\delta_{f}=20^{\circ}$ flowfield to burst mode excitation. Re $\mathbf{e}_{c}=$ $\mathbf{0 . 2 5} \times 10^{6}, \alpha=6^{\circ}$. 
into the interaction of the perturbations introduced at the flap shoulder with the separating shear layer for flap deflection angles $\left(\delta_{f}\right)$ of $20^{\circ}$ and $40^{\circ}$. The surface pressure data and PIV data will aid in determining the appropriate location and type of actuation to add to the existing actuators available for controlling separation on the trailing edge flap of the model. Preliminary results presented of the transients associated with separation and attachment of the flow to the trailing edge flap will be useful for upcoming closed-loop control active separation control research.

\section{Acknowledgments}

The authors would like to thank the Subsonic Fixed Wing program for funding the research and the following individuals for their support Anthony Washburn, Catherine McGinley, Norman Schaeffler, Steve Wilkinson, Greg Jones, Luther Jenkins, Charlie Debro, and Marie Lane.

\section{References}

\footnotetext{
${ }^{1}$ Seifert, A., Darabi, A., and Wygnanski, I., "On the Delay of Airfoil Stall by Periodic Excitation," Journal of Aircraft, Vol. 33, No. 4, 1996, pp. 691-699.

${ }^{2}$ Seifert, A. and Pack, L. G., "Oscillatory Control of Separation at High Reynolds Numbers," AIAA Journal, Vol. 37, No. 9, 1999, pp. 1062-1071.

${ }^{3}$ Greenblatt, D. and Wygnanski, I., "The Control of Flow Separation by Periodic Excitation," Progress in Aerospace Sciences, Vol. 36, 2004, pp. 487-545.

${ }^{4}$ Seifert, A., Greenblatt, D., and Wygnanski, I., "Active Separation Control: an Overview of Reynolds and Mach Number Effects," Aerospace Science and Technology, Vol. 8, 2004, pp. 569-582.

${ }^{5}$ Melton, L. P., Yao, C. S., and Seifert, A., "Application of Excitation from Multiple Locations on a Simplified High-Lift System," AIAA Paper 2004-2324, June 2004.

${ }^{6}$ Melton, L. P., Schaeffler, N. W., and Lin, J. C., "High-Lift System for a Supercritical Airfoil: Simplified by Active Flow Control," AIAA Paper 2007-0707, Jan. 2007.

${ }^{7}$ Greenblatt, D., "Dual Location Separation Control on a Semispan Wing," AIAA Journal, Vol. 45, No. 8, 2007, pp. 1848-1860.

${ }^{8}$ Greenblatt, D. and Washburn, A., "Influence of Finite Span and Sweep on Active Flow Control Efficacy," AIAA Journal, Vol. 46, No. 7, 2008, pp. 1675-1694.

${ }^{9}$ Greenblatt, D., "Management of Vortices Trailing Flapped Wings via Separation Control," AIAA Paper 2005-0061, Jan. 2005.

${ }^{10}$ King, R. A. and Breuer, K. A., "Acoustic Receptivity of a Blasius Boundary Layer with 2-D and Oblique Surface Waviness," AIAA Paper 2000-2538, June 2000.

${ }^{11}$ Jones, G. S., Viken, S. A., Washburn, A. E., Jenkins, L. N., and Cagle, C. M., "An Active Flow Circulation Controlled Flap Concept for General Aviation Aircraft Applications," AIAA Paper 2002-3157, June 2002.

${ }^{12}$ Barlow, J. B., W. H. Rae, J., and Pope, A., Low Speed Wind Tunnel Testing, John Wiley and Son, 3rd ed., 1999.

${ }^{13}$ Youngren, H., Kroninger, C., Chang, M., and Jameson, S., "Low Reynolds Number Testing of the AG38 Airfoil for the SAMARAI Nano Air Vehicle," AIAA Paper 2008-417, Jan. 2008.

${ }^{14}$ Coleman, H. W. and Steele, W. G., Experimentation and Uncertainty Analysis for Engineers, John Wiley and Son, 2nd ed., 1999.

${ }^{15}$ Greenblatt, D., Nishri, B., Darabi, A., and Wygnanski, I., "Some Factors Affecting Stall Control with Particular Emphasis on Dynamic Stall," AIAA Paper 1999-3504, June 1999.

${ }^{16}$ Melton, L. P., Yao, C., and Seifert, A., "Active Control of Separation from the Flap of a Supercritical Airfoil," AIAA Journal, Vol. 44, No. 1, 2006, pp. 34-41, previously AIAA paper 2003-4005.

${ }^{17}$ Wygnanski, I., "The Variables Affecting the Control of Separation by Periodic Excitation," AIAA Paper 2004-2505, June 2004.

${ }^{18}$ Seifert, A. and Pack, L. G., "Active Flow Separation Control on Wall-Mounted Hump at High Reynolds Numbers," AIAA Journal, Vol. 40, No. 7, 2002, pp. 1363-1372.

${ }^{19}$ Darabi, A. and Wygnanski, I., "Active Management of Naturally Separated Flow Over a Solid Surface. Part 1. The Forced Reattachment Process," Journal of Fluid Mechanics, Vol. 510, 2004, pp. 105-129.

${ }^{20}$ Darabi, A. and Wygnanski, I., "Active Management of Naturally Separated Flow Over a Solid Surface. Part 2. The Separation Process," Journal of Fluid Mechanics, Vol. 510, 2004, pp. 131-144.
} 